\title{
Heterogeneous and Homogenous Multistabilities in a Novel 4D Memristor-Based Chaotic System with Discrete Bifurcation Diagrams
}

\author{
Lilian Huang ${ }^{(D)}$, Wenju Yao, Jianhong Xiang $(D$, and Zefeng Zhang \\ College of Information and Communication Engineering, Harbin Engineering University, Harbin 150001, China \\ Correspondence should be addressed to Jianhong Xiang; xiangjianhong@hrbeu.edu.cn
}

Received 25 December 2019; Revised 20 February 2020; Accepted 11 March 2020; Published 25 April 2020

Guest Editor: Viet-Thanh Pham

Copyright (c) 2020 Lilian Huang et al. This is an open access article distributed under the Creative Commons Attribution License, which permits unrestricted use, distribution, and reproduction in any medium, provided the original work is properly cited.

\begin{abstract}
In this paper, a new 4D memristor-based chaotic system is constructed by using a smooth flux-controlled memristor to replace a resistor in the realization circuit of a 3D chaotic system. Compared with general chaotic systems, the chaotic system can generate coexisting infinitely many attractors. The proposed chaotic system not only possesses heterogeneous multistability but also possesses homogenous multistability. When the parameters of system are fixed, the chaotic system only generates two kinds of chaotic attractors with different positions in a very large range of initial values. Different from other chaotic systems with continuous bifurcation diagrams, this system has discrete bifurcation diagrams when the initial values change. In addition, this paper reveals the relationship between the symmetry of coexisting attractors and the symmetry of initial values in the system. The dynamic behaviors of the new system are analyzed by equilibrium point and stability, bifurcation diagrams, Lyapunov exponents, and phase orbit diagrams. Finally, the chaotic attractors are captured through circuit simulation, which verifies numerical simulation.
\end{abstract}

\section{Introduction}

Memristor was first proposed by Chua [1] in 1971 and is the fourth basic electronic component manufactured by HP Labs in 2008 [2]. The discovery of memristors has caused an upsurge in studying and applying memristors. Due to the nonlinearity of memristor, it has been applied in many fields, such as flash memory [2,3], neuromorphic computing [4, 5], neural network [6,7], and chaotic system [8-11] based on chaos synchronization for encryption algorithms [12, 13] and secure communication $[14,15]$.

Memristor is a nonlinear element, and its resistance depends on the voltage or current signal, so it has been widely used in the construction of chaotic circuits in recent years [16-19]. In 2008, Itoh and Chua proposed together a Chua's chaotic circuit based on memristor. The dynamic analysis results show that Chua's chaotic circuit based on memristors has more complex dynamic characteristics than classic Chua's chaotic circuit [17]. In 2010, a Chua's chaotic circuit based on memristance was proposed by replacing Chua's diode with a smooth flux-controlled memristor and a negative conductance [18]. In 2017, a multiscroll hyperchaotic system was proposed by introducing the memristor into the jerk multiscroll system, and the numbers of scrolls can be controlled by adjusting the coefficient before the term related to memristor [19].

In recent years, multistability [20-25] and extreme multistability [26-32] have become research hotspots in the field of chaotic systems. Multistability means that when the system parameters remain unchanged, the system can generate more than one attractor with different initial values. When the number of attractors is infinite, this phenomenon is called extreme multistability. Coexisting attractors and hidden coexisting attractors are shown in a memristive system with many equilibrium points in reference [23]. A wing-variable chaotic system with coexisting twin-wing attractors is proposed by replacing one of the resistors of the pseudo-four-wing chaotic system with a memristor in 
reference [24]. In the same year, a memristor-based chaotic system is constructed by introducing an ideal flux-controlled memristor with absolute value nonlinearity into an existing hypogenetic chaotic jerk system, which can exhibit the extreme multistability phenomenon in reference [31]. A simplest third-order memristive chaotic system with hidden attractors is proposed, which exhibits the extreme multistability phenomenon of coexisting infinitely many attractors in reference [32].

Although multistability and extreme multistability in memristive chaotic systems had been reported in many existing papers, most of them researched the heterogeneous multistability of chaotic systems, and homogenous multistability was rarely reported. Heterogeneous multistability means that under the same parameters, the chaotic system has some chaotic attractors with different structures, while homogenous multistability means the chaotic system can generate attractors with the same structure, but the amplitudes and positions of their attractors can be different. In this article, the mathematical model of a memristor is employed to construct the chaotic system owning heterogeneous and homogenous multistabilities. Besides, the presented memristor-based system displays other complex dynamic characteristics, including constant Lyapunov exponents, discrete bifurcation diagrams, the symmetry of coexisting attractors, and so on.

The rest of this paper is organized as follows. In Section 2 , a new chaotic system based on the model of a memristor is studied. And basic properties of the proposed system are investigated, including symmetry and dissipation, equilibrium, and stability. In Section 3, complex dynamic behaviors of the memristor-based chaotic system are analyzed. In Section 4, extreme multistability of the chaotic system is investigated by bifurcation diagrams and Lyapunov exponent spectra, and the dynamic analysis results show that the chaotic system possesses not only heterogeneous multistability but also homogenous multistability. In Section 5, the presented chaotic system is realized by analog circuit and the experimental results are given. Finally, some conclusions are drawn.

\section{Basic Properties of the Memristive Chaotic System}

A 3D pseudo-four-wing chaotic system was proposed by Liu and Chen [33,34]. Actually, it is a coexisting two-wing system, and it can be described as follows:

$$
\left\{\begin{array}{l}
\dot{x}=a x-b y z, \\
\dot{y}=-c y+x z, \\
\dot{z}=-d z+x y
\end{array}\right.
$$

where $a, b, c$, and $d$ are all constants and $x, y$, and $z$ are the state variables.

By utilizing a smooth flux-controlled memristor to substitute a resistor in realization circuit of system (1), a novel $4 \mathrm{D}$ memristor-based chaotic system is given by

$$
\left\{\begin{array}{l}
\dot{x}=a x-b y z, \\
\dot{y}=-c y+x z+e W(w) x, \\
\dot{z}=-\mathrm{d} z+x y, \\
\dot{w}=h x
\end{array}\right.
$$

where $e$ and $h$ are positive parameters and $W(w)$ is a memductance function.

The memductance function $W(w)$ is shown as

$$
W(w)=f+3 g w^{2},
$$

where $f$ and $g$ are two positive constants and $w$ is the state variable.

2.1. Symmetry and Dissipativity. The symmetry property of chaotic systems is an important property. The memristive chaotic system (2) is invariant if we do the transformation $(x, y, z, w) \longrightarrow(-x,-y, z,-w)$, which means system (2) has to be symmetric with respect to $z$ axis in state space.

The dissipativity of system (2) is expressed by the following formula:

$$
\nabla V=\frac{\partial \dot{x}}{\partial x}+\frac{\partial \dot{y}}{\partial y}+\frac{\partial \dot{z}}{\partial z}+\frac{\partial \dot{w}}{\partial w}=a-c-d .
$$

When $a, c$, and $d$ satisfy condition $a-c-d<0$, the system is dissipative. It means that the volume of phase space will be contracted to zero in exponential form $e^{-(a-c-d)}$ and all trajectories of the system are confined to zero volume.

2.2. Equilibria and Stability. Let the terms on left-hand side of system (2) be zero, and we can easily observe that the system has a line equilibrium

$$
O=\{(x, y, z, w) \mid x=y=z=0, w=k\},
$$

where $k$ is any real constant.

By linearizing system (2) at point $O$, we can obtain the Jacobian matrix of the equation on $O$.

$$
J_{o}=\left(\begin{array}{cccc}
a & 0 & 0 & 0 \\
e W(k) & -c & 0 & 0 \\
0 & 0 & -d & 0 \\
h & 0 & 0 & 0
\end{array}\right) .
$$

According to the Jacobian matrix (6), the characteristic equation can be obtained as follows:

$$
\lambda(\lambda-a)(\lambda+c)(\lambda+d)=0 .
$$

We can solve its eigenvalues easily, and they can be expressed by

$$
\begin{aligned}
& \lambda_{1}=0, \\
& \lambda_{2}=a, \\
& \lambda_{3}=-c, \\
& \lambda_{4}=-d .
\end{aligned}
$$


The values of $a, c$, and $d$ are all positive, so $\lambda_{3}$ and $\lambda_{4}$ are always negative, and $\lambda_{2}$ is always positive. Therefore, system (2) has an unstable saddle point.

\section{Dynamics of the Memristor-Based Chaotic System}

3.1. Phase Portraits and Lyapunov Exponents. The Lyapunov exponent spectra are an effective way of judging whether the system has chaotic behavior. The main characteristics of nonlinear dynamical systems can be described by the number of positive Lyapunov exponents. When the system has only one Lyapunov exponent greater than zero, the system has chaotic dynamic behavior. When a nonlinear system has more than two or equal to two Lyapunov exponents greater than zero, the system has hyperchaotic dynamic behavior.

When the parameters of the chaotic system are set as $a=4, b=6, c=20, d=5, e=0.01, f=1,3 g=0.1$, and $h=$ 0.1 and the initial conditions are set to $(1,1,0,0)$, system (2) can generate chaotic attractor as shown in Figure 1. The corresponding Lyapunov exponents are computed as 0.5161, $-0.0104,-0.0645$, and -21.5665 , and the Lyapunov dimension $\mathrm{dL}=3.0204$, which indicates the system has chaotic behavior.

3.2. Poincaré Projection. The Poincaré projection is another effective way of judging whether the system has chaotic behaviour. Take projections $x=0, y=0, z=6$, and $w=1.4$, respectively, and the system obtains the Poincaré projections as shown in Figure 2. A large area of points can be observed in these pictures, which indicate that the system has chaotic behaviour.

\section{Extreme Multistability in the Memristor- Based Chaotic System}

4.1. Dynamic Analysis of Heterogeneous Multistability. Heterogeneous multistability means under the same parameters, a system can generate several or even infinitely many coexisting attractors with different structures, while homogenous multistability means that a chaotic system can generate the same structure coexisting attractors but with different positions or amplitudes.

In system (2), when the parameters are set as $a=4$, $b=6, c=20, d=5, e=0.01, f=1,3 g=0.1$, and $h=0.1$ and initial conditions are set as $(1,0,0, w(0))$, the system can generate various coexisting attractors depending on $w(0)$. The typical chaotic attractors are shown in Figure 3. Besides, system (2) can generate other kinds of coexisting attractors, coexisting limit cycle attractors, and symmetric limit cycle attractor as shown in Figures 4, 5, and 6, respectively.

When $w(0)$ is changed in the region $[-50,50]$, the bifurcation diagram of the state variable $w$ and Lyapunov exponent spectra are shown in Figures $7(a)$ and 7(b), respectively. As shown in Figure 7(a), the bifurcation diagram of the state variable $w$ is almost linear. It can be seen from Figure $7(\mathrm{~b})$ that the chaotic attractor with a positive
Lyapunov exponent is mainly located at the region $[-36,-32],[-22,22]$, and $[32,35]$ (the last Lyapunov exponent is not displayed because it is always a big negative number). And system (2) can also generate other kinds of attractors and limit cycle attractors, which means the system has heterogeneous multistability.

4.2. Dynamic Analysis of Homogenous Multistability. The parameters of system (2) remain unchanged, and the initial conditions are set as $(x(0), 1,0,0)$. When $x(0)$ is varied in the region $\left[-10^{4}, 10^{4}\right]$, the bifurcation diagrams of the state variable $z$, the state variable $w$, and its Lyapunov exponent spectra are plotted in Figures 8(a), 8(b), and 8(c), respectively. Besides, when $x(0)$ is varied in the region $[-0.1,0.1]$, Lyapunov exponent spectra are plotted in Figure $8(\mathrm{~d})$.

It can be seen from Figure 8(a) that when the initial condition $x(0)$ is varied in the region $\left[-10^{4}, 10^{4}\right]$, the state variable $z$ shows two kinds of steady chaotic states. When $x(0)$ is varied in the region $\left[-10^{4}, 0\right)$, state variable $z$ is located below or above the $z$ axis, while $x(0)$ is varied in the region $\left(0,10^{4}\right]$, the state variable $z$ is all located above the $z$ axis. From Figure $8(\mathrm{~b})$, when the initial condition $x(0)$ is varied in the region $\left[-10^{4}, 10^{4}\right]$, there are many discrete small line segments in bifurcation diagram of the state variable $w$, which means the state variable $w$ exists in infinite steady chaotic states. And this phenomenon also indicates the system can generate coexisting infinitely many attractors, which means system (2) has the extreme multistability. Different from other systems possessing extreme multistability, system (2) only generates two kinds of chaotic attractors with different positions in a very large range of initial values, which are distributed along the $w$ axis parallelly. As shown in Figure 8(c), it is obvious that the four Lyapunov exponents are always approximately constant and the largest Lyapunov exponent is always positive except for the zero point when $x(0)$ is varied in the region $\left[-10^{4}, 10^{4}\right]$, which means system (2) can exhibit chaotic behavior except zero point. Figure $8(\mathrm{~d})$ shows that the region cannot exhibit chaotic behavior which is very small.

Considering the particularity of system (2), it is necessary to discuss the system when $x(0)=1$. The control parameters of system (2) remain unchanged, and the initial conditions are set as $(0,1,0,0)$. The LEs are $4.0002,-0.2034$, -5.0005 , and -19.8272 , which means system (2) cannot exhibit chaotic behavior under this circumstance.

It has been confirmed that there are coexisting infinitely many attractors in chaotic system (2) according to the above analysis. When $x(0)$ is set to $10,-10,50,+50,100,-100$, and 1 , respectively, the phase portraits of coexisting infinitely many attractors in the $x-w$ plane, the $y-w$ plane, the $z-$ $w$ plane, and the $w-z-x$ space are shown in Figures 9(a), 9(b), 9(c), and 9(d), respectively. Figure 9 clearly reveals the coexistence of a large number of same attractors with different positions, which implies the emergence of homogenous multistability. This result of the phase portraits is consistent with the bifurcation diagrams and Lyapunov spectrum with respect to initial condition $x(0)$. 


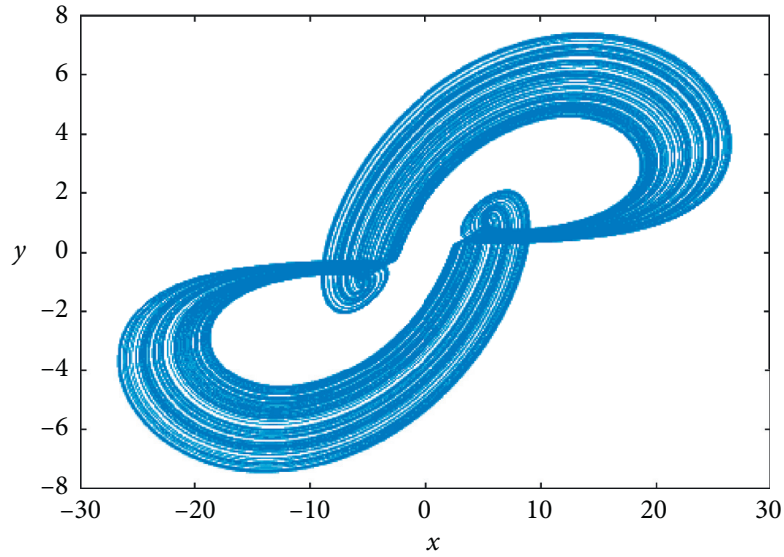

(a)

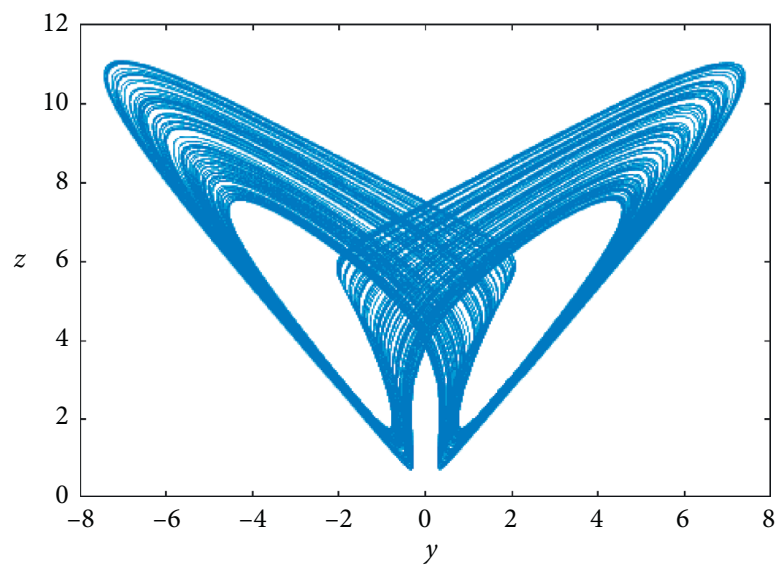

(c)

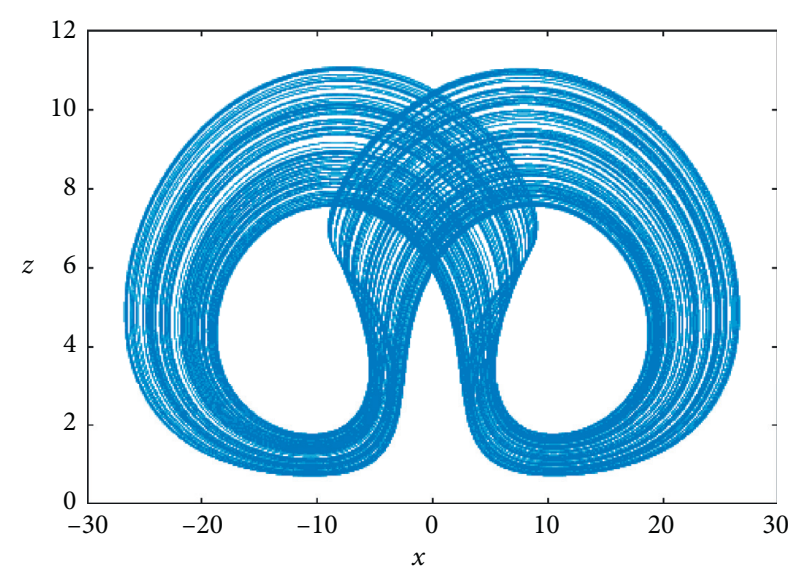

(b)

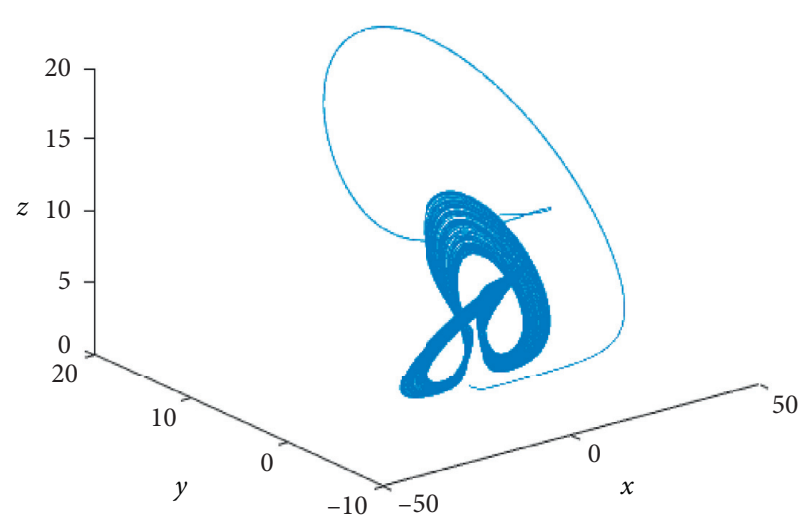

(d)

Figure 1: Phase portraits of system (2) when $a=4, b=6, c=20, d=5, e=0.01, f=1,3 g=0.1$, and $h=0.1$ : (a) projection on $x-y$ plane, (b) projection on $x-z$ plane, (c) projection on $y-z$ plane, and (d) $3 \mathrm{D}$ view in the $x-y-z$ space.

The parameters of system (2) are set as $a=4, b=6$, $c=20, d=5, e=0.01, f=1,3 g=0.1$, and $h=0.1$, the initial conditions are set as $(1, y(0), 0,0)$, and the initial condition $y(0)$ is used as the independent variable of bifurcation diagram. When $y(0)$ is changed in the region $\left[-10^{4}, 10^{4}\right]$, the bifurcation diagram of the state variable $w$ and its Lyapunov exponent spectra are plotted in Figures 10(a) and 10(b), respectively. As can be seen from Figure 10(a), when the initial condition $y(0)$ is changed in the region $\left[-10^{4}, 10^{4}\right]$, there are many discrete small line segments in bifurcation diagram of the state variable $w$, which implies that there are coexisting infinitely many attractors in system (2). It can be clearly seen from Figure 10(b) that four Lyapunov exponents are always approximately constant and largest Lyapunov exponent is always positive when is $y(0)$ changed in the region $\left[-10^{4}, 10^{4}\right]$, which means system (2) only exhibits chaotic behavior.

The parameters of system (2) are set as $a=4, b=6$, $c=20, d=5, e=0.01, f=1,3 g=0.1$, and $h=0.1$, the initial conditions are set as $(1,0, z(0), 0)$, and the initial condition $z(0)$ is used as the independent variable of bifurcation diagram. When $z(0)$ is changed in the region $\left[-10^{4}, 10^{4}\right]$, the bifurcation diagram of the state variable $w$ and its Lyapunov exponent spectra are plotted in Figures 11(a) and 11(b), respectively. The bifurcation diagram of the state variable $w$ and its Lyapunov exponent spectra are similar with those with the initial conditions set as $(1, y(0), 0,0)$. Many discrete small line segments are more closely clustered in the bifurcation diagram of the state variable $w$ for the initial conditions set as $(1,0, z(0), 0)$ compared with the one for the initial conditions set as $(1, y(0), 0,0)$. And Lyapunov exponent spectra for the initial conditions set as $(1,0, z(0), 0)$ are similar with the ones for the initial conditions set as $(1, y(0), 0,0)$, and four Lyapunov exponents are always approximately constant and largest Lyapunov exponent is always positive when $z(0)$ is changed in the region $\left[-10^{4}, 10^{4}\right]$.

\subsection{Symmetry in Infinitely Many Coexisting Attractors.} Obviously, system (2) is invariant if we do the transformation $(x, y, z, w) \longrightarrow(-x,-y, z,-w)$, which means that $(x, y, z, w)$ and $(-x,-y, z,-w)$ are all solutions to the equation of the system. This symmetry characteristic of system (2) could be served to explain the presence of symmetric coexisting attractors in state space. 


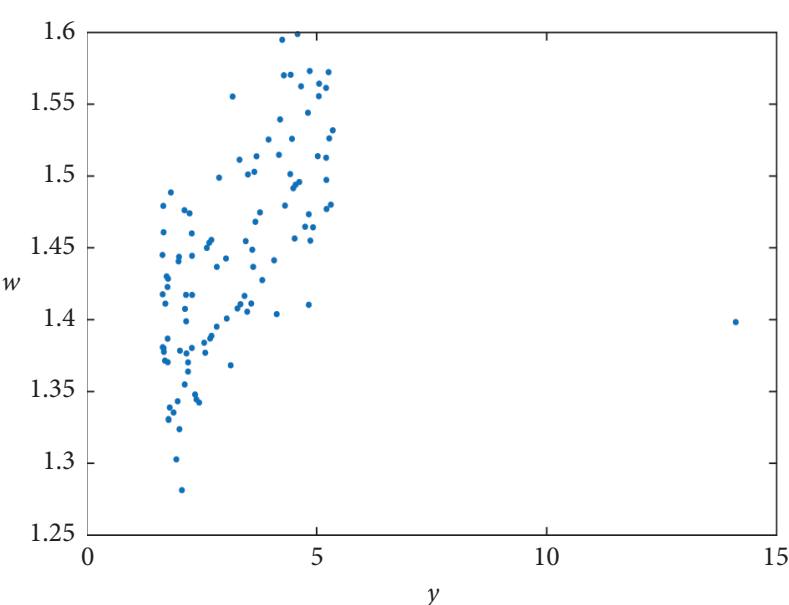

(a)

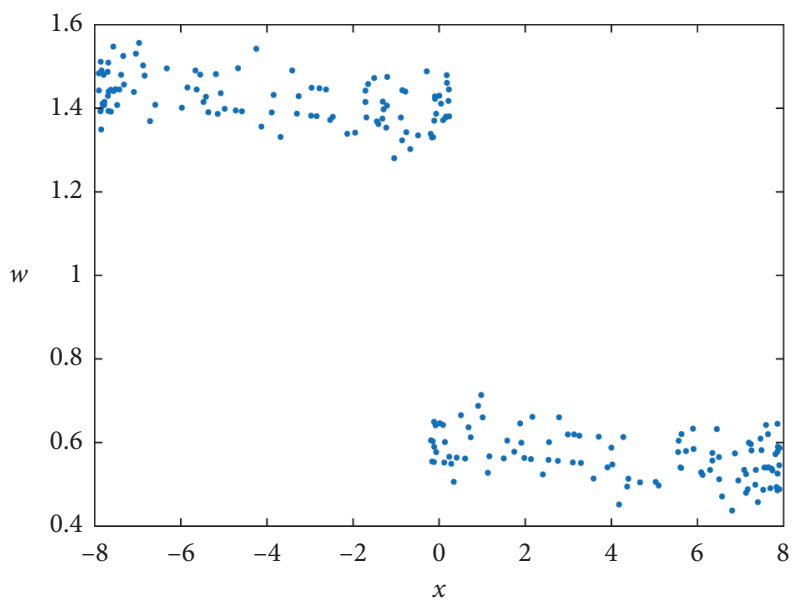

(c)

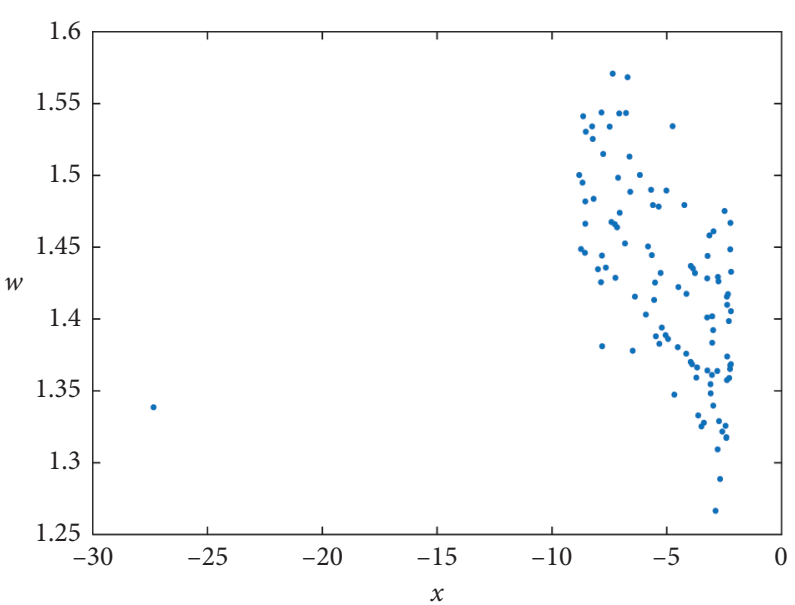

(b)

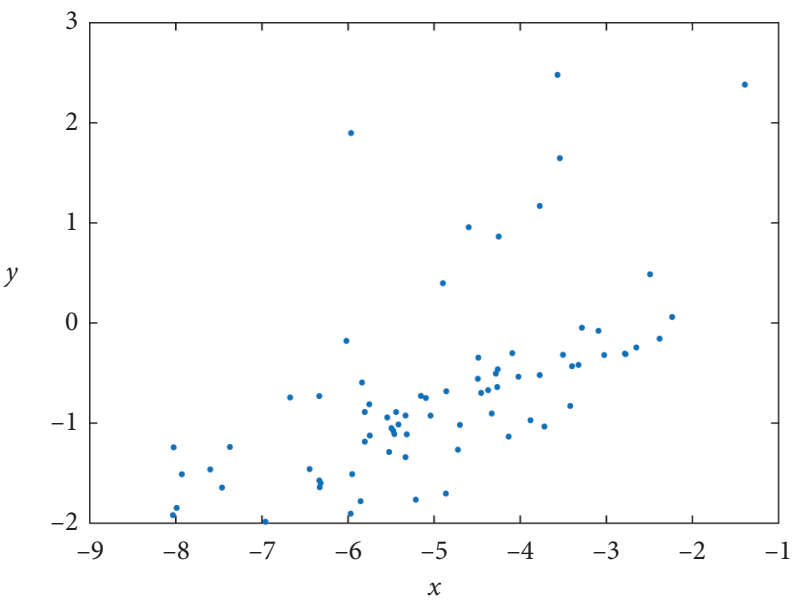

(d)

Figure 2: Poincaré sections of system (2): (a) projection on $y-w$ plane when Poincaré section is $x=0$, (b) projection on $x-w$ plane when Poincaré section is $y=0,(\mathrm{c})$ projection on $x-w$ plane when Poincaré section is $z=6$, and (d) projection on $x-y$ plane when Poincaré section is $w=1.4$.

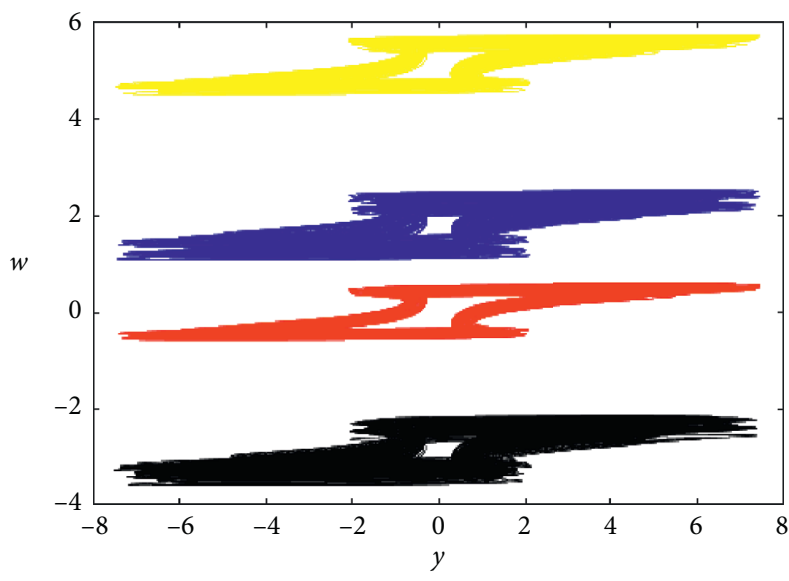

(a)

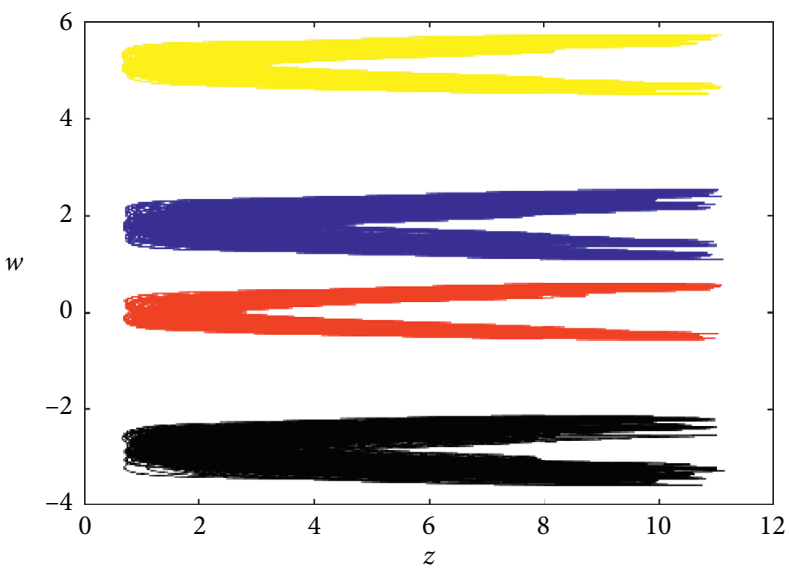

(b)

FIgURE 3: Phase portraits of coexisting infinitely many attractors in (a) $y-w$ and (b) planes (the blue one starts from initial conditions $(1,0,0,1)$, the red one starts from $(1,0,0,-1)$, the yellow one starts from $(1,0,0,4)$, and the black one starts from $(1,0,0,-4))$. 


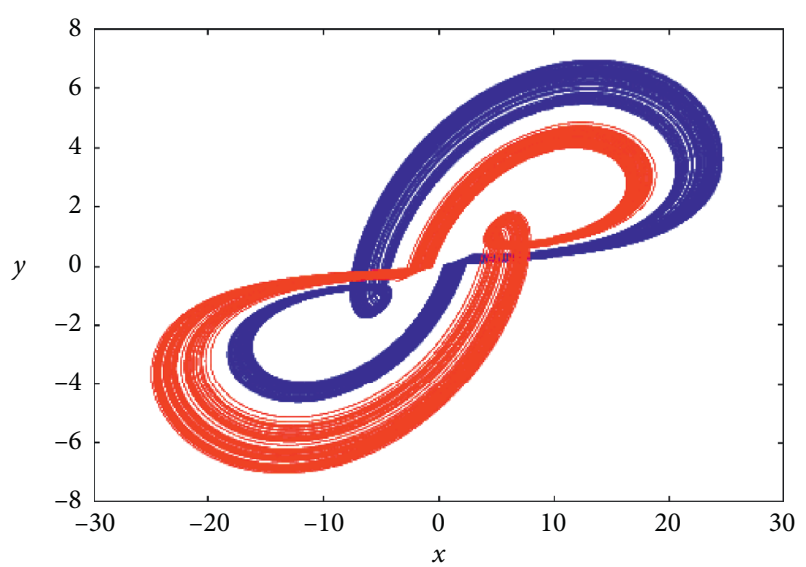

(a)

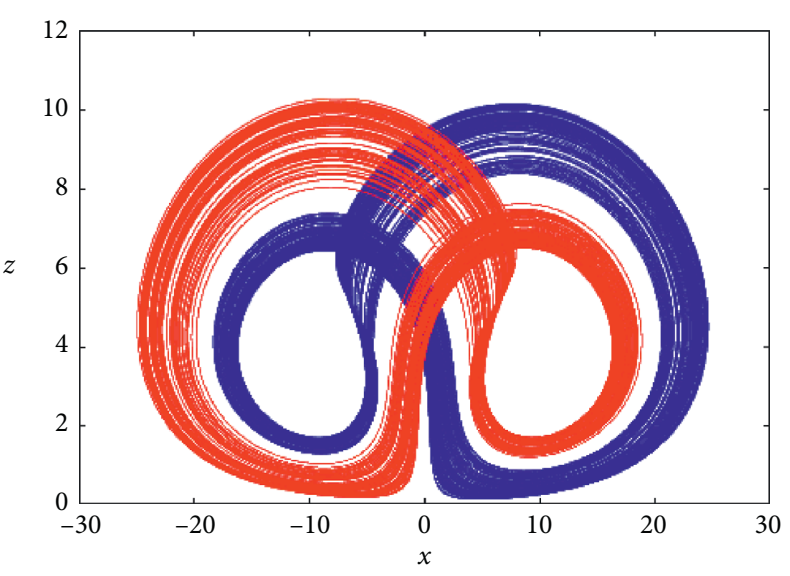

(b)

Figure 4: Phase portraits of coexisting attractors in (a) $x-y$ and (b) $x-z$ planes (the blue one starts from initial conditions $(1,0,0,22)$ and the red one starts from $(1,0,0,-22))$.

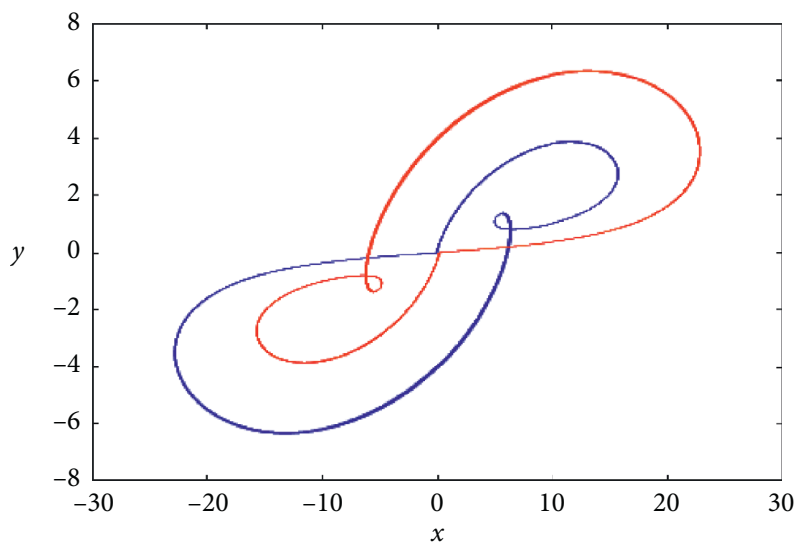

(a)

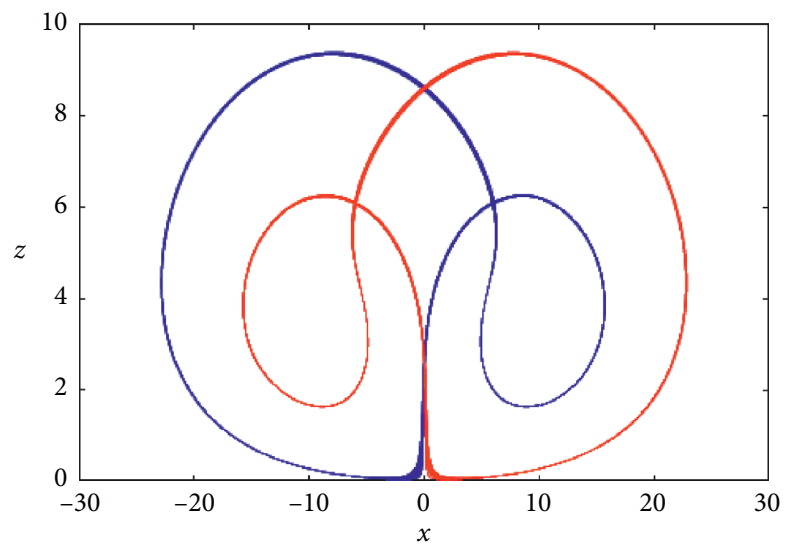

(b)

FIGURE 5: Phase portraits of coexisting limit cycle attractors in (a) $x-y$ and (b) $x-z$ planes (the blue one starts from initial conditions $(1,0,0,26)$ and the red one starts from $(1,0,0,-26))$.

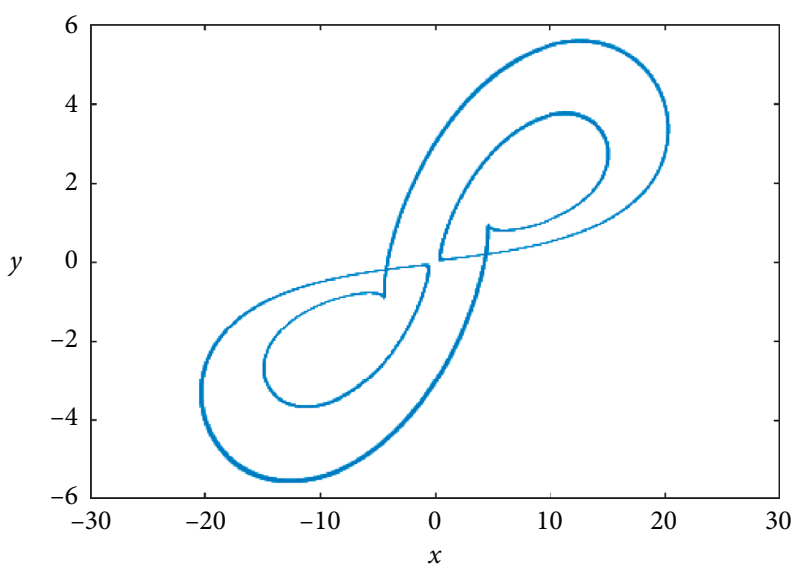

(a)

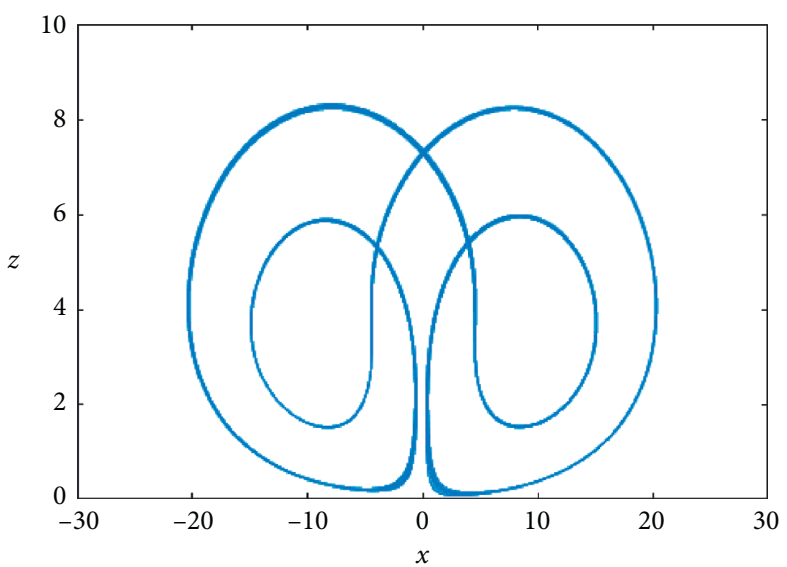

(b)

Figure 6: Phase portraits of limit cycle in (a) $x-y$ and (b) $x-z$ planes for initial conditions set as $(1,0,0,30)$. 


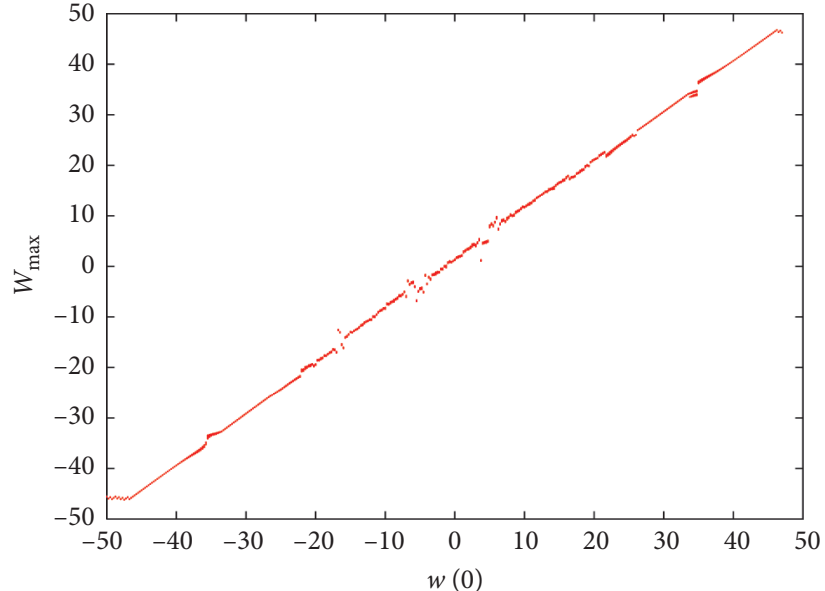

(a)

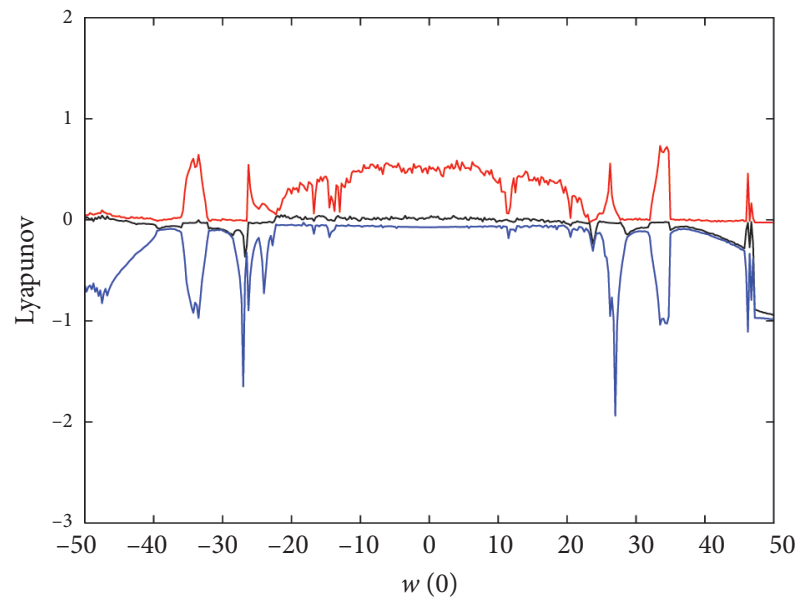

(b)

Figure 7: (a) Bifurcation diagram of the state variable $w$ and (b) Lyapunov spectrum with respect to memristor initial condition $w(0)$.

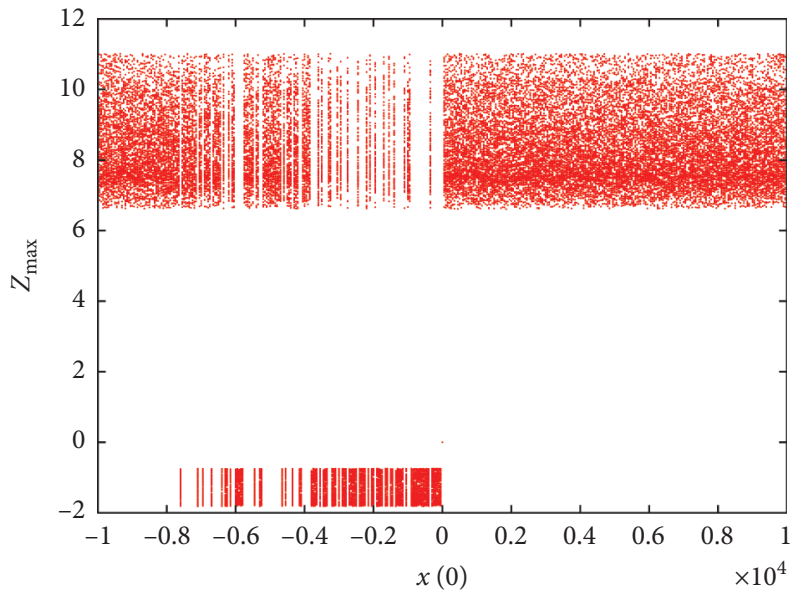

(a)

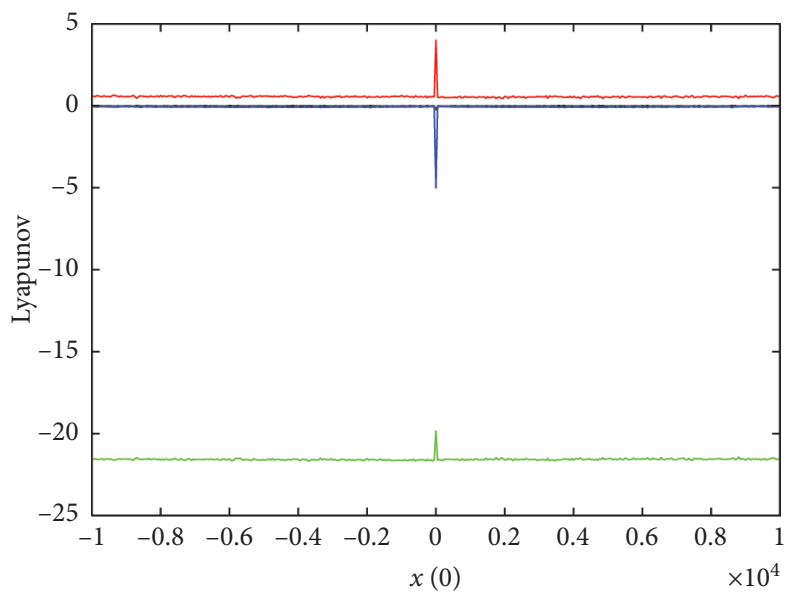

(c)

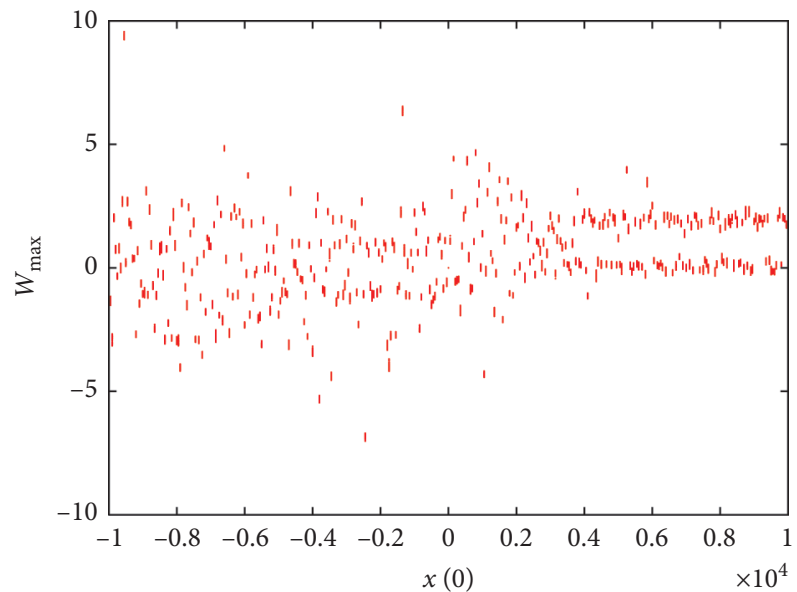

(b)

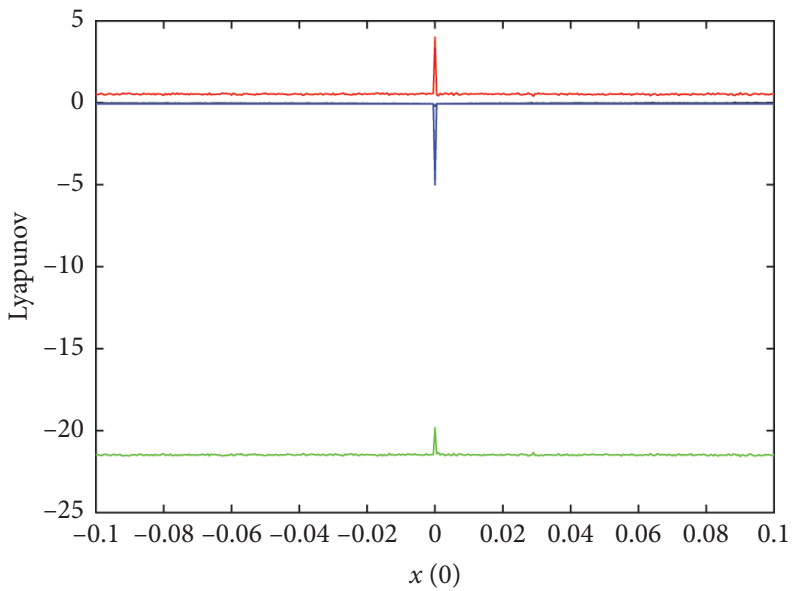

(d)

Figure 8: (a) Bifurcation diagram of the state variable $z$, (b) bifurcation diagram of the state variable $w$, (c) Lyapunov spectrum with respect to initial condition $x(0)$ in the region $\left[-10^{4}, 10^{4}\right]$, and (d) Lyapunov spectrum with respect to initial condition $x(0)$ in the region $[-0.1,0.1]$. 


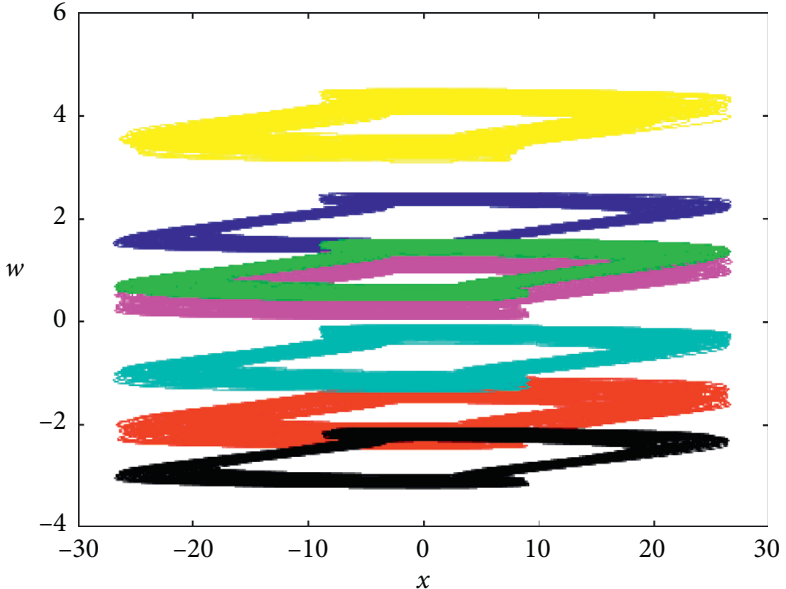

(a)

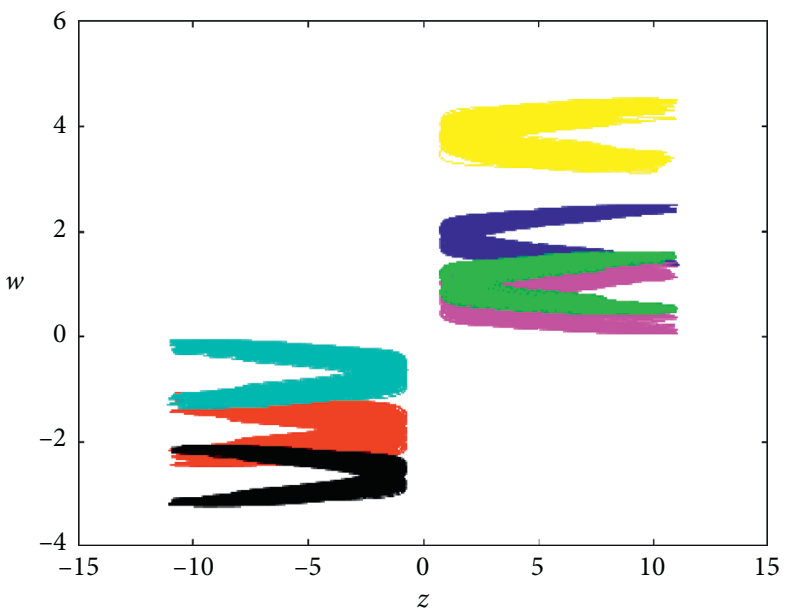

(c)

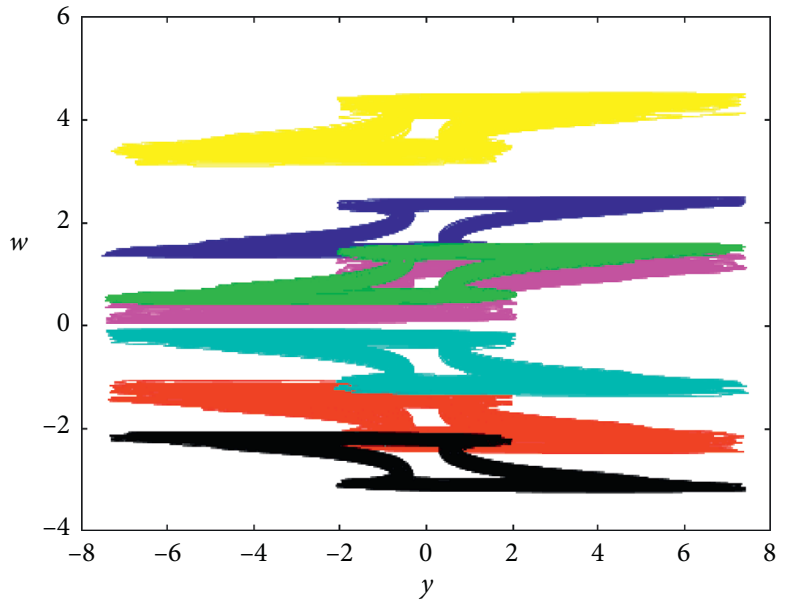

(b)

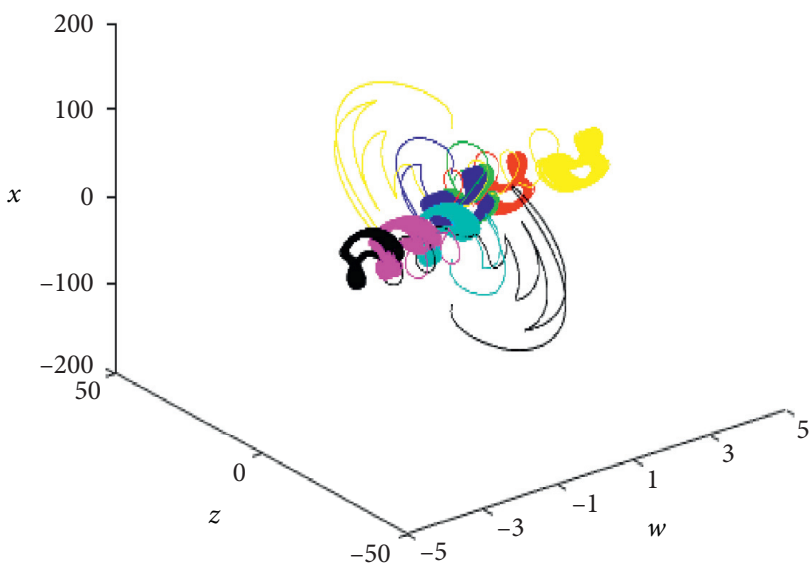

(d)

FigURE 9: Phase portraits of coexisting infinitely many attractors on (a) $x-w$ plane, (b) $y-w$ plane, (c) $z-w$ plane, and (d) $w-z-x$ plane in chaotic system (2) (the blue one starts from initial conditions $(10,1,0,0)$, the red one starts from $(-10,1,0,0)$, the pink one starts from $(50,1,0,0)$, the cyan one starts from $(-50,1,0,0)$, the yellow one starts from $(100,1,0,0)$, the black one starts from $(-100,1,0,0)$, and the green one starts from $(1,1,0,0))$.

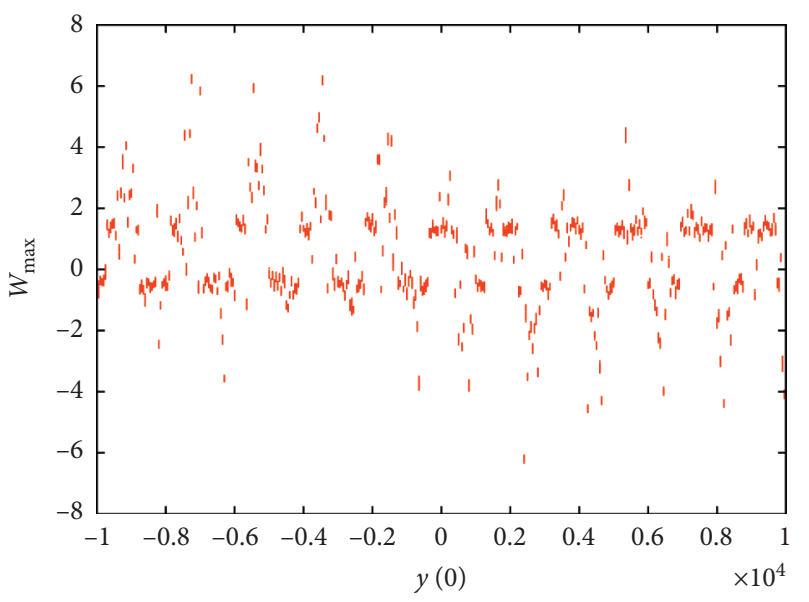

(a)

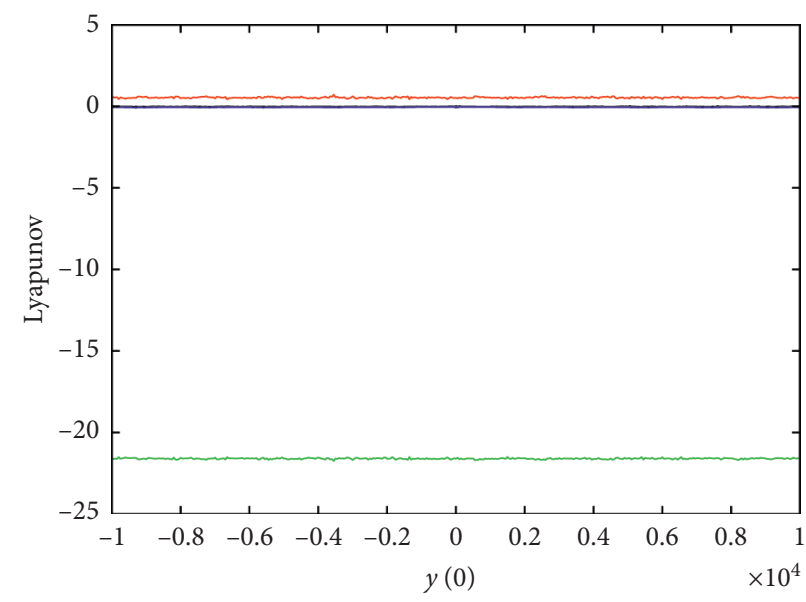

(b)

Figure 10: (a) Bifurcation diagram of the state variable $w$ and (b) Lyapunov spectrum with respect to initial condition $y(0)$ in the region $\left[-10^{4}, 10^{4}\right]$. 


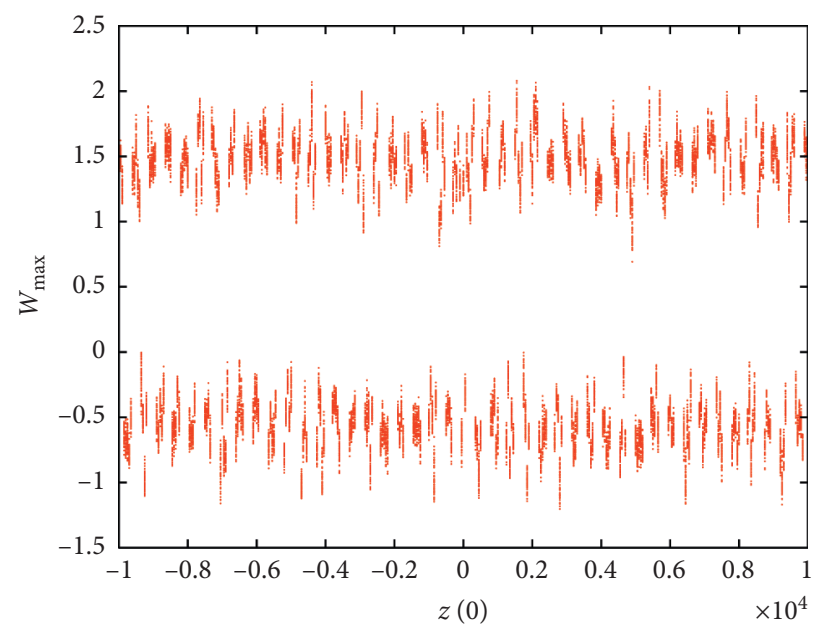

(a)

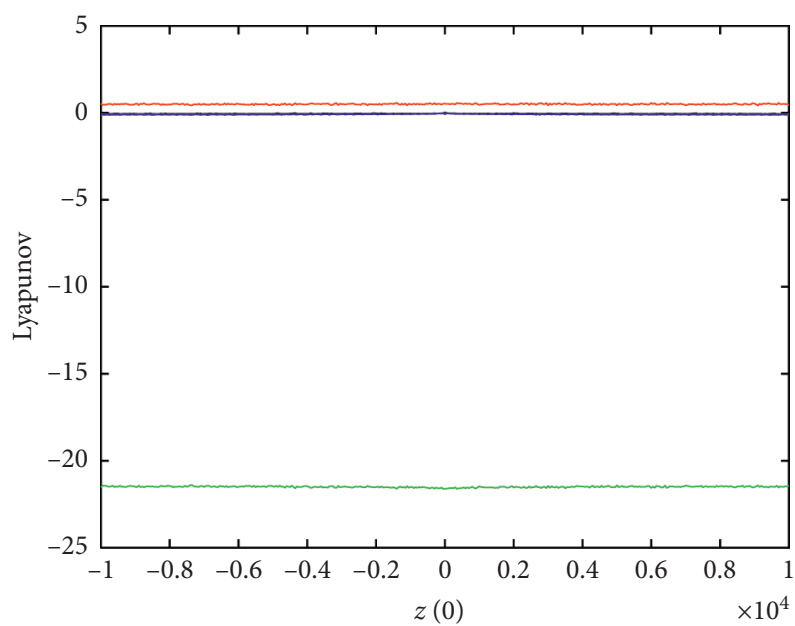

(b)

FigURE 11: (a) Bifurcation diagram of the state variable $w$ and (b) Lyapunov spectrum with respect to initial condition $z(0)$ in the region $\left[-10^{4}, 10^{4}\right]$.

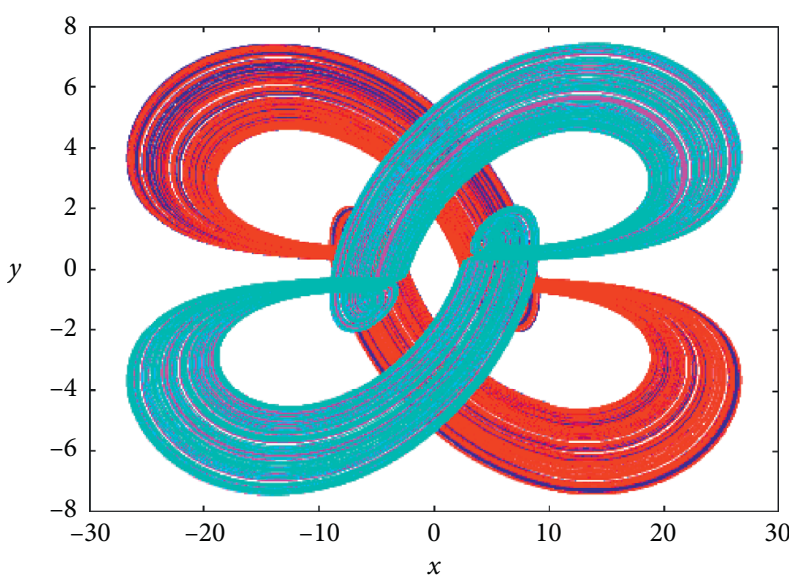

(a)

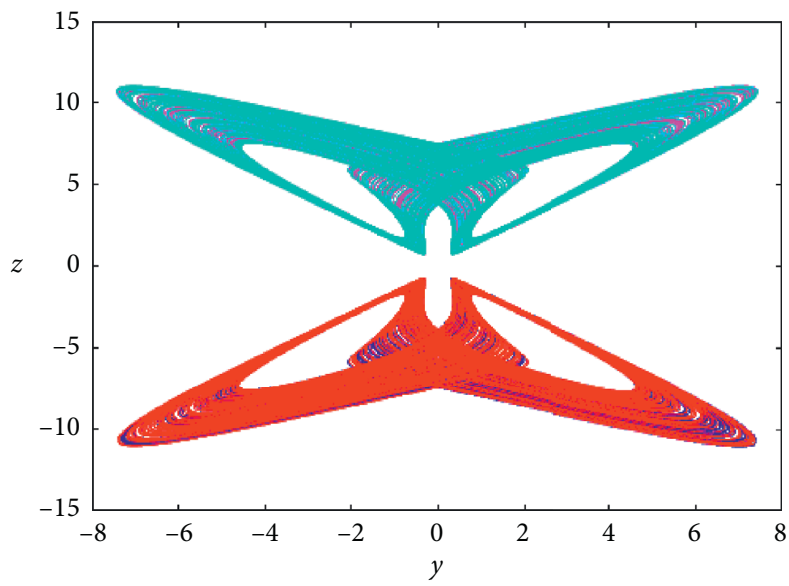

(c)

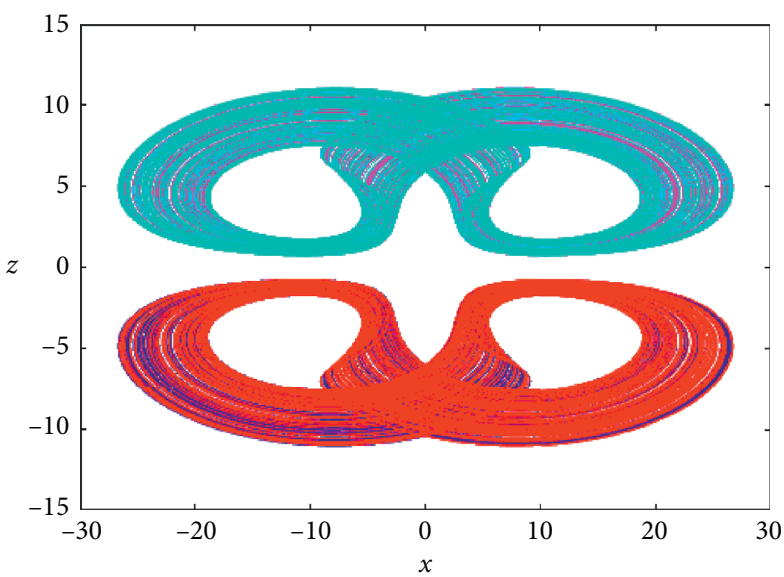

(b)

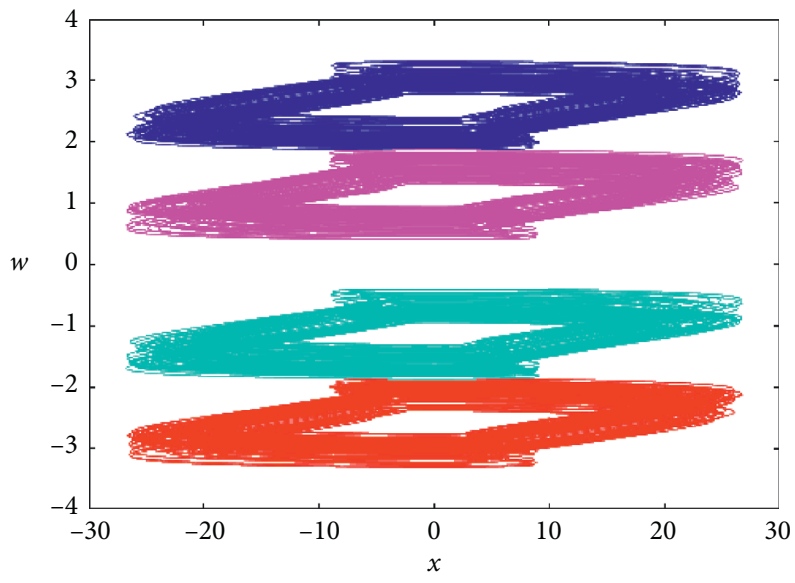

(d)

FIGURE 12: Continued. 


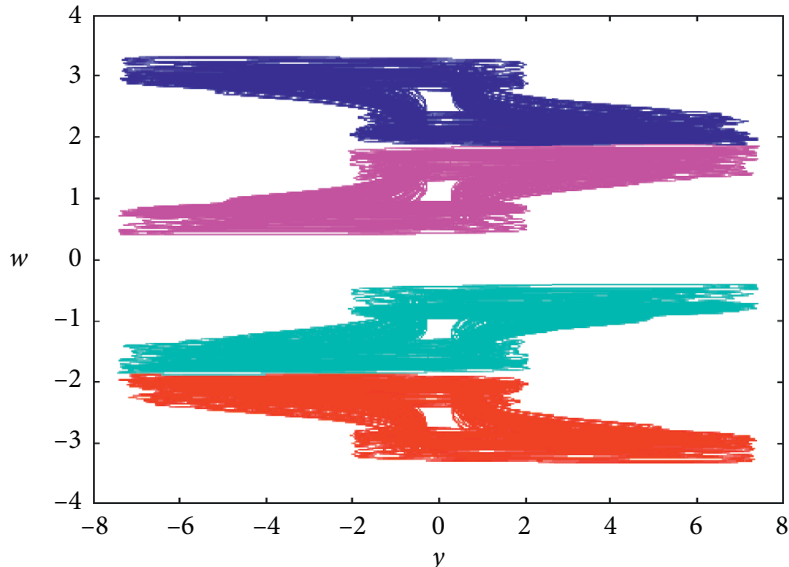

(e)

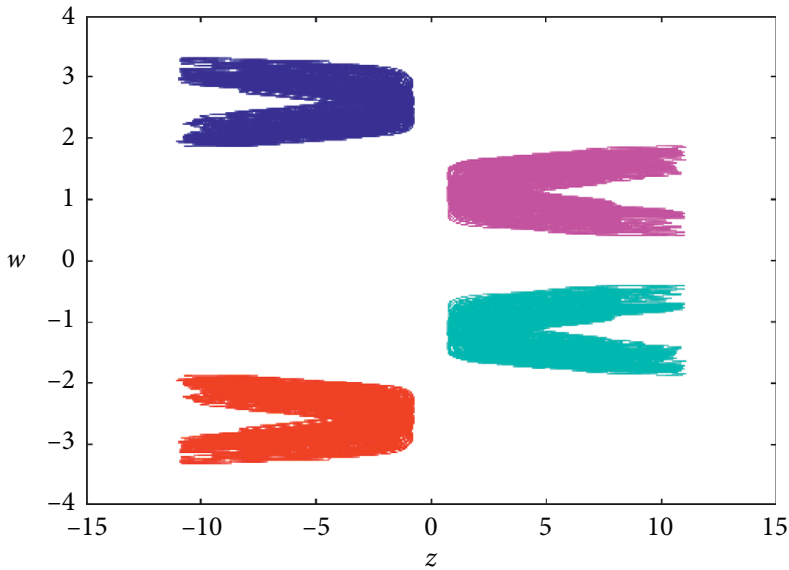

(f)

Figure 12: Phase portraits of symmetric coexisting infinitely many attractors in chaotic system (2) (the blue one starts from initial conditions $(1,1,-10,1)$, the red one starts from $(-1,-1,-10,-1)$, the pink one starts from $(10,20,15,2)$, and the cyan one starts from $(-10,-20,15,-2))$.

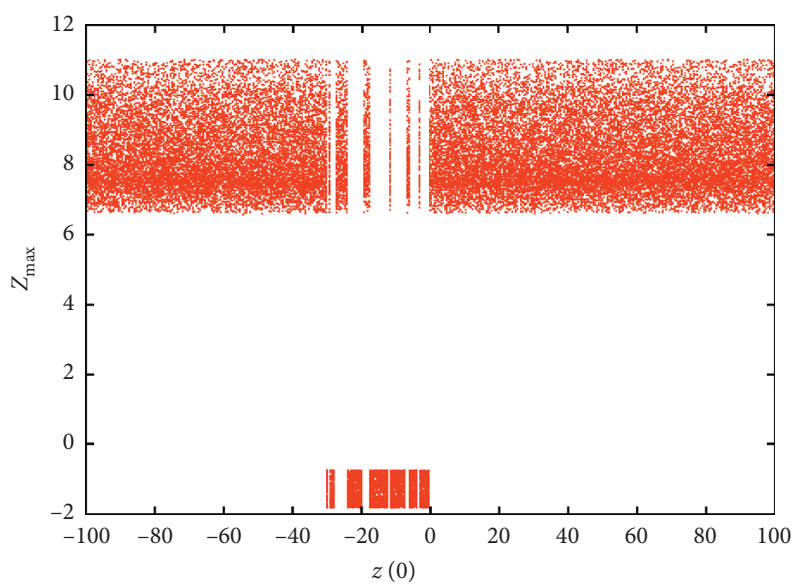

(a)

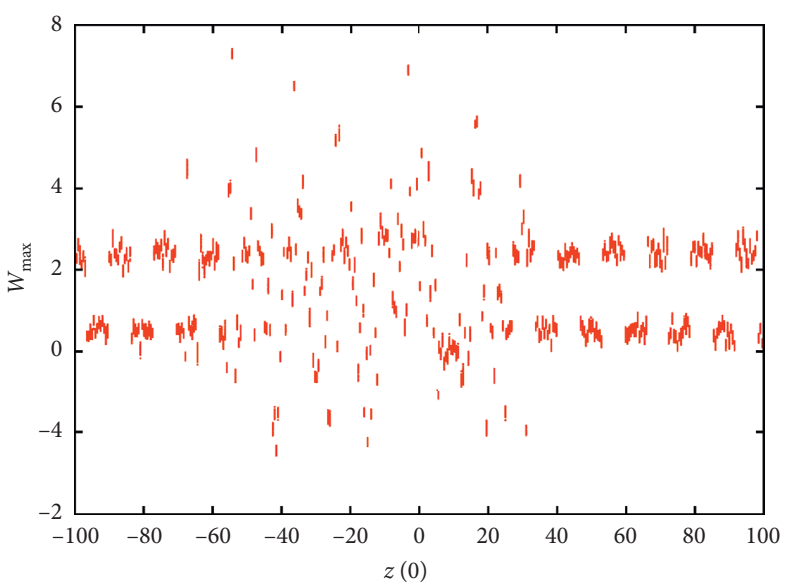

(b)

FIgURE 13: (a) Bifurcation diagram of the state variable $z$ and (b) bifurcation diagram of the state variable $w$ with respect to initial conditions $(1,1, z(0), 1)$.

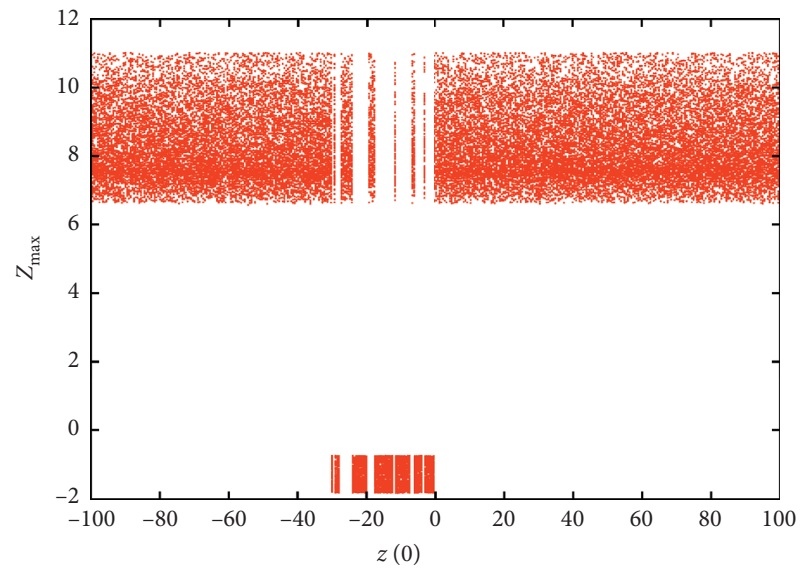

(a)

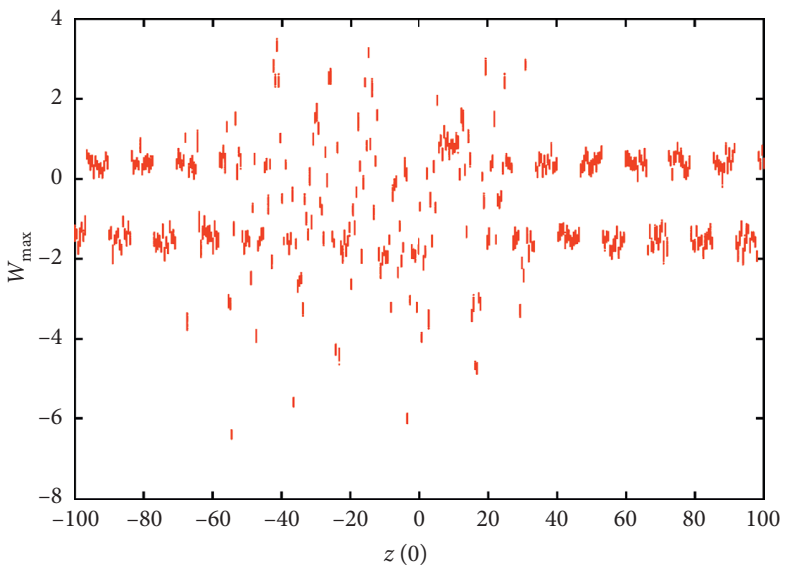

(b)

FIGURE 14: (a) Bifurcation diagram of the state variable $z$ and (b) bifurcation diagram of the state variable $w$ with respect to initial conditions $(-1,-1, z(0),-1)$. 

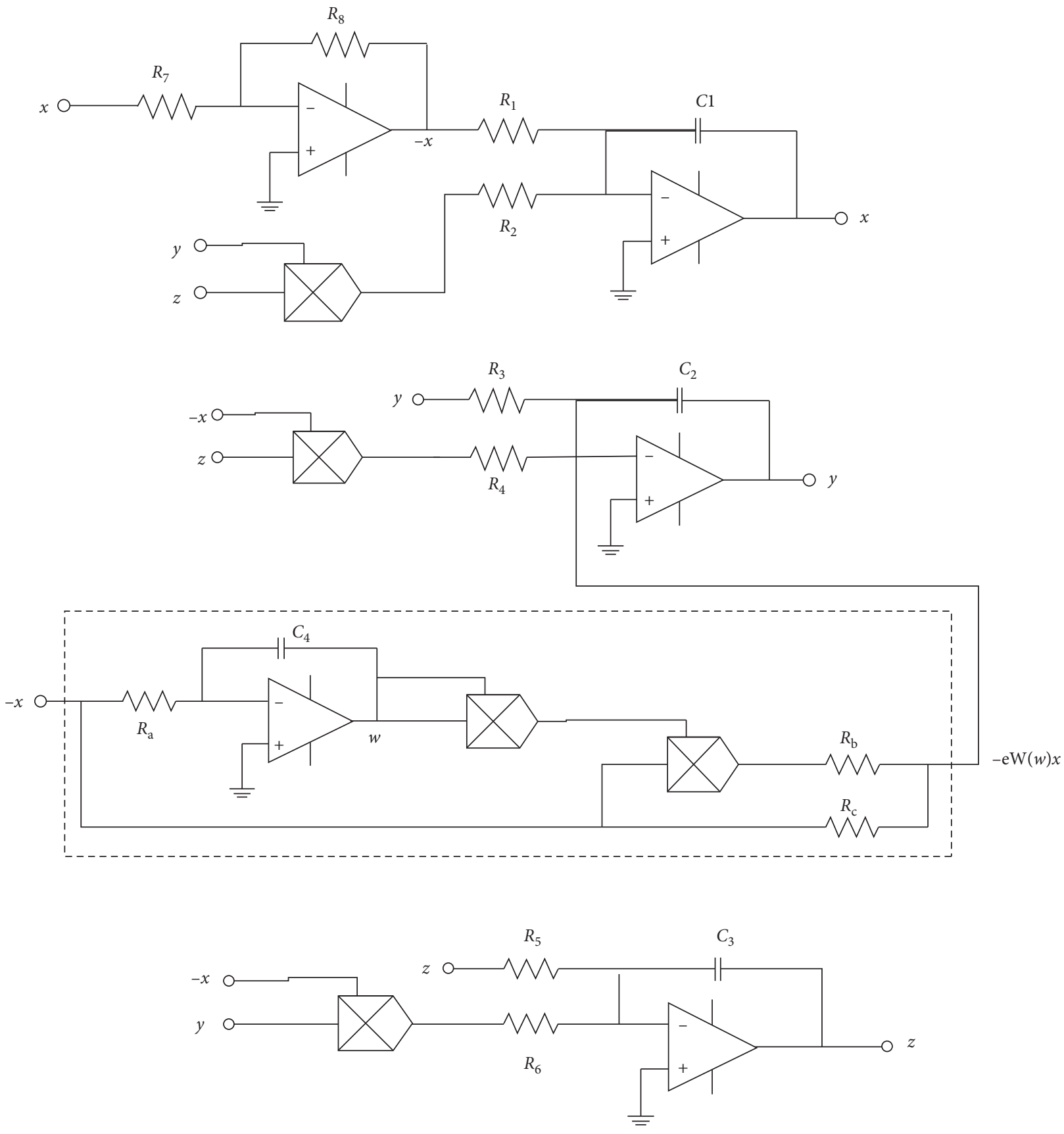

Figure 15: Circuit diagram of memristive system (2).

If we set parameters as $a=4, b=6, c=20, d=5$, $e=0.01, f=1,3 g=0.1$, and $h=0.1$, the system can generate many pairs of symmetric coexisting attractors for the corresponding conditions set as $(x(0), y(0), z(0), w(0))$ and $(-x(0),-y(0), z(0),-w(0))$. And two pairs of symmetric coexisting attractors of these are shown in Figure 12, where the blue one and the red one are a pair of symmetric attractors and the pink one and the cyan one are another pair of symmetric attractors. The projections of the symmetric attractors on the coordinate planes can be shown in the form of central symmetry or axial symmetry. In system (2), it is centrosymmetric on the $x-y$ plane, the $x-w$ plane, and the $y-w$ plane and axisymmetric on the $x-z$ plane, the $y-z$ plane, and the $z-w$ plane. It is worth noting that the structures of many coexisting attractors in Figure 9 are the same roughly, and there are differences in the details. But the structures of each pairs of coexisting attractors in Figure 12 are symmetric exactly.

In order to further verify symmetry of coexisting infinitely many attractors in chaotic system (2), bifurcation diagrams of the state variable $z$ and the state variable $w$ are given. The parameters of system (2) are assigned as $a=4$, $b=6, c=20, d=5, e=0.01, f=0.1,3 g=0.1$, and $h=0.1$, and the initial conditions are set as $(1,1, z(0), 1)$, and the initial condition $z(0)$ is used as the independent variable of bifurcation diagram. When $z(0)$ is varied in the region 


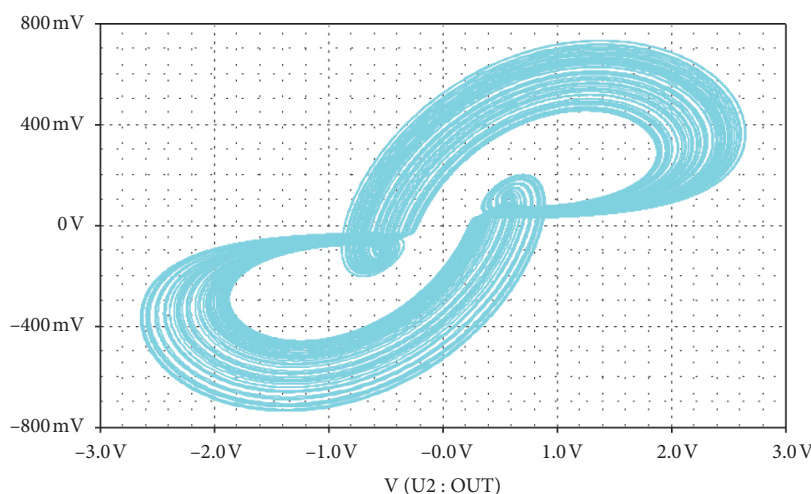

V (U3 : OUT)

(a)

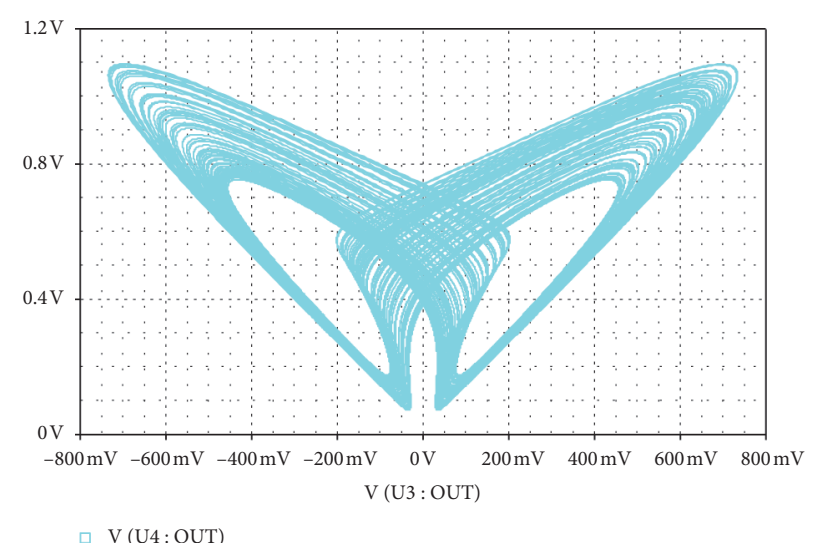

(c)

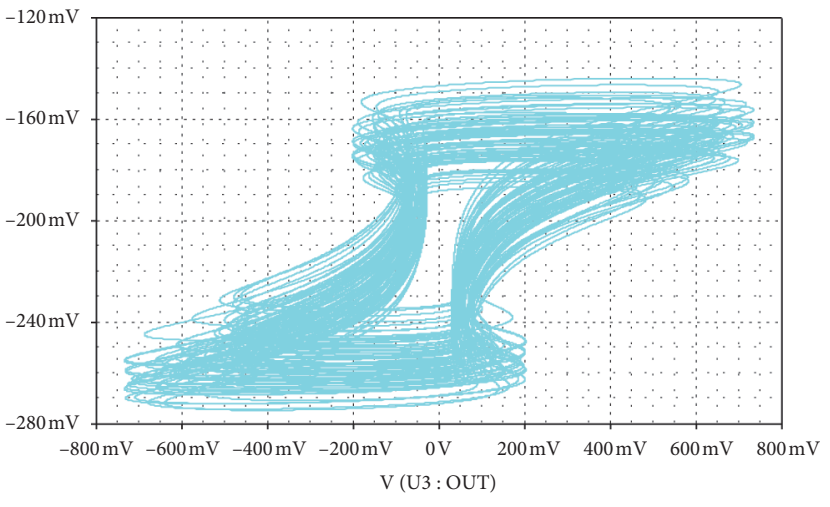

V(U5: OUT)

(e)

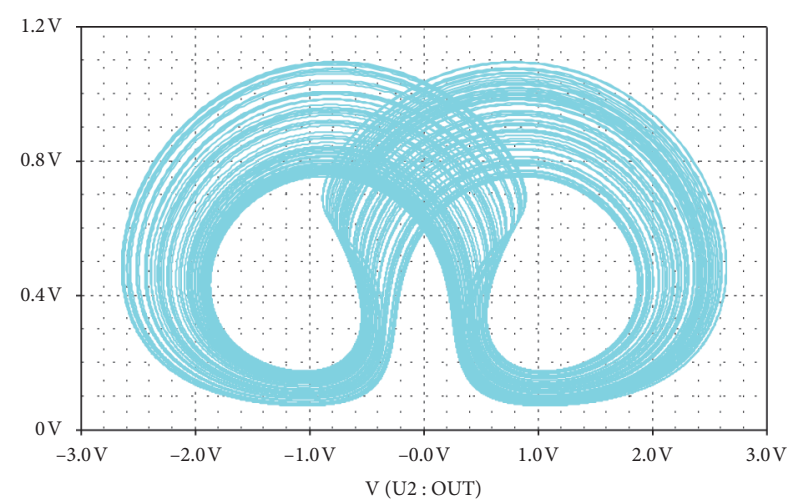

$\checkmark \mathrm{V}(\mathrm{U} 4$ : OUT $)$

(b)

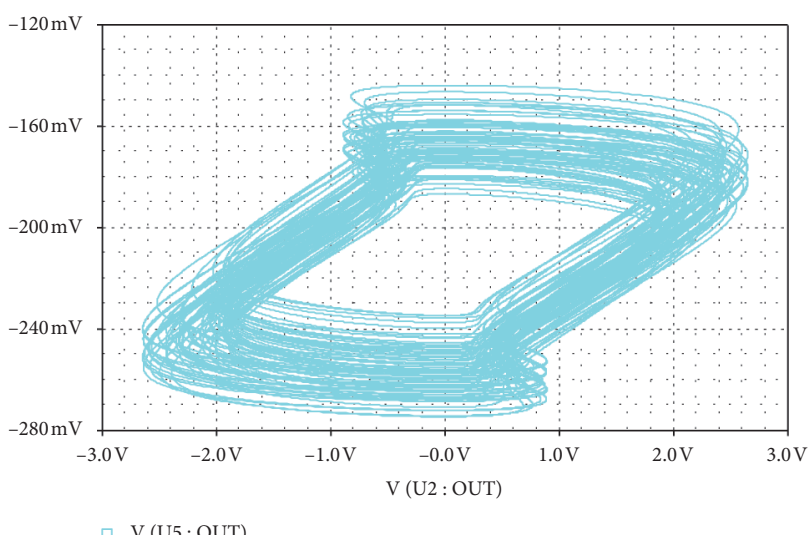

(d)

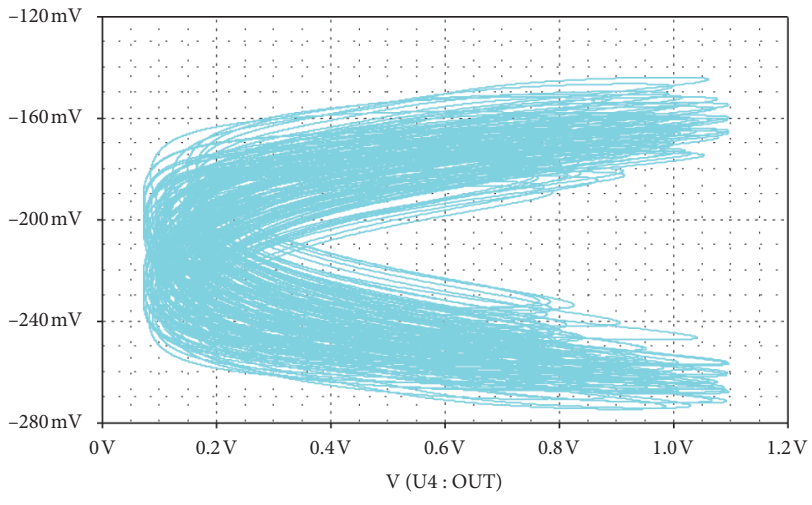

V (U5 : OUT)

(f)

FIgURE 16: PSpice simulated phase portraits of chaotic attractors with initial voltages $(1 \mathrm{~V}, 0.1 \mathrm{~V}, 0 \mathrm{~V}, 0 \mathrm{~V})$.

$[-100,100]$, the bifurcation diagrams of the state variable $z$ and the state variable $w$ are plotted in Figures 13(a) and 13(b), respectively. Similarly, the parameters of system (2) remain unchanged, the initial conditions are set as $(-1,-1, z(0),-1)$, and the initial condition $z(0)$ is used as the independent variable. When $z(0)$ is varied in the region $[100,-100]$, the bifurcation diagrams of the state variable $z$ and the state variable $w$ are plotted in Figures 14(a) and 14(b), respectively.
A comparison of the Figures 13(a) and 14(a) indicates that bifurcation diagrams of the state variable $z$ for the initial conditions set as $(1,1, z(0), 1)$ and $(-1,-1, z(0),-1)$ are the same exactly. And a comparison of the Figures 13(b) and 14(b) shows that they are symmetric about the horizontal axis. This phenomenon reflects the fact that when the parameters of system (2) set as $a=4, b=6, c=20, d=5$, $e=0.01, f=1,3 g=0.1, h=0.1$, the structures of coexisting attractors with conditions set as $(1,1, z(0), 1)$ and 


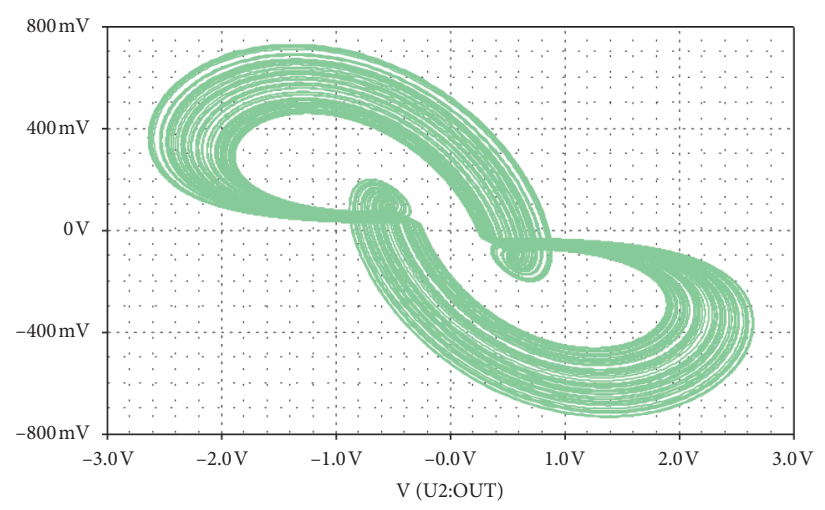

V (U3:OUT)

(a)

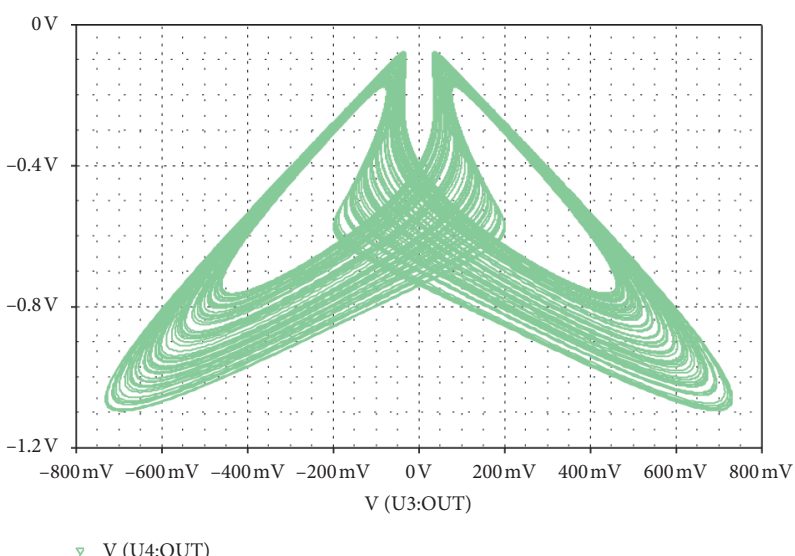

(c)

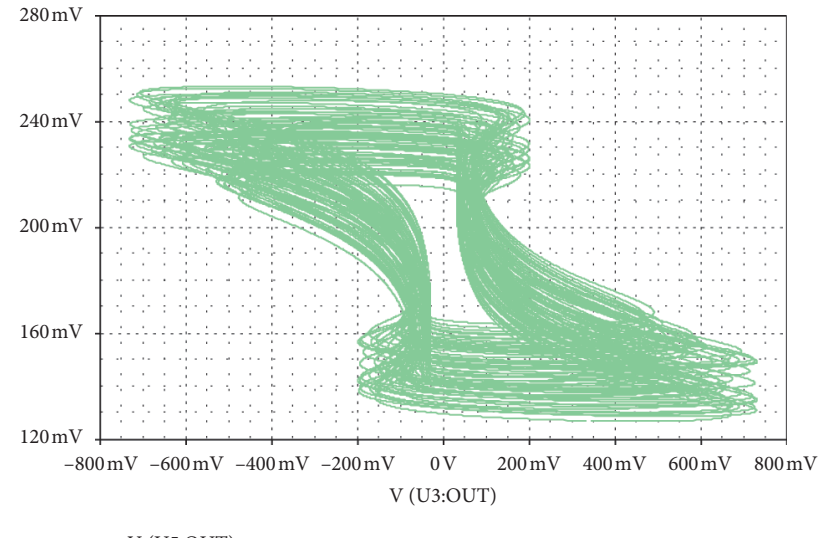

(e)

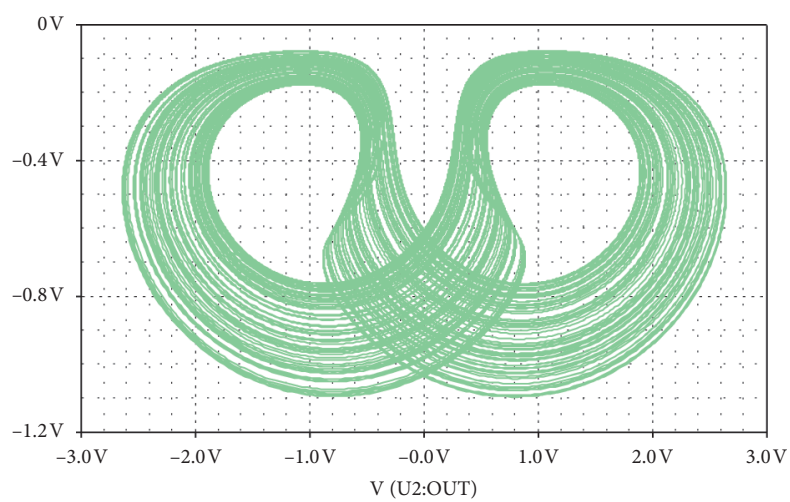

V (U4:OUT)

(b)

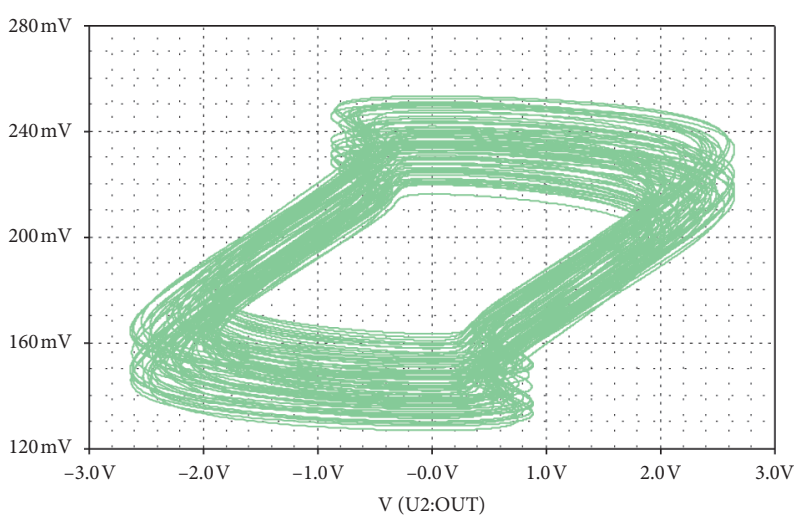

V $($ U5:OUT)

(d)

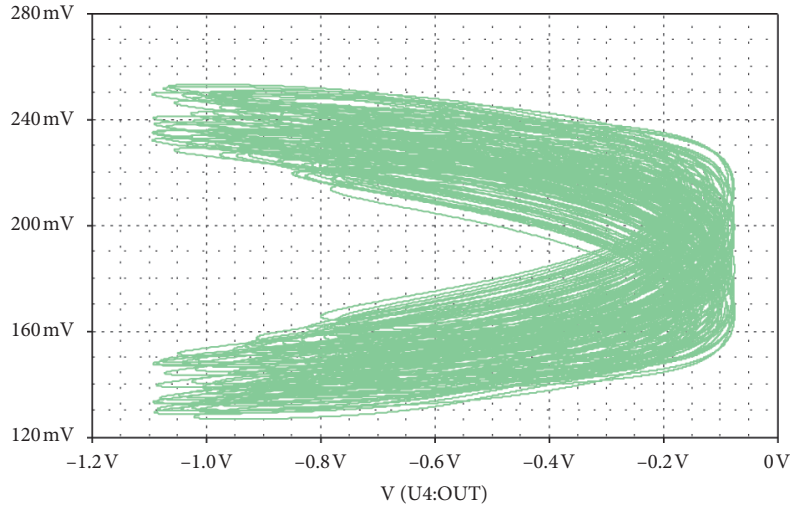

$\checkmark \mathrm{V}(\mathrm{U} 5: \mathrm{OUT})$

(f)

FIGURE 17: PSpice simulated phase portraits of chaotic attractors with initial voltages $(-1 \mathrm{~V}, 0.1 \mathrm{~V}, 0 \mathrm{~V}, 0 \mathrm{~V})$.

$(-1,-1, z(0),-1)$ are symmetric with respect to $z$ coordinate axis.

We compared the advantages and disadvantages of this system with other improved Liu-and-Chen systems in the literature [11, 23, 24]. The system proposed in reference [11] is a three-dimensional chaotic system without memristor. And it can generate three-scroll and four-scroll chaotic attractors. The system in reference [23] is a four-dimensional chaotic system with two memristors, which can generate various kinds of attractors and hidden attractors. The system in reference [24] is a four-dimensional chaotic system with a memristor, which can generate three-wing, four-wing, and coexisting two-wing chaotic attractors. It has line equilibrium points. This system has a line equilibrium, within which the attractors generated are hidden. The new system is a four-dimensional chaotic system with a memristor, which can generate coexisting infinitely many attractors distributed along the $w$ axis parallelly. It produces hidden attractors 
because it has a line equilibrium. In addition, the system has discrete bifurcation diagrams and many symmetrical coexisting attractors. Compared with above improved Liuand-Chen systems, we can find that chaotic systems with memristor have more complex dynamic behaviors than chaotic systems without memristor and are more likely to have extreme multistability.

\section{Circuit Implementation}

In this section, complex dynamic behaviors of the proposed chaotic system can be observed by analog circuit, where integrated operational amplifiers and multipliers are used to construct the circuit for generating chaotic attractors. Supply voltages of operational amplifiers are $E= \pm 15 \mathrm{~V}$. The input and output range of all the multipliers is between $-15 \mathrm{~V}$ to $15 \mathrm{~V}$. However, the values of the state variables $x, y, z$, and $w$ may be out of this range. Thus, it is necessary that state variables $x, y, z$, and $w$ are compressed to the $1 / 10$ of original system to be limited in the region of $(-15 \mathrm{~V}, 15 \mathrm{~V})$, which is the reference voltage of the operational amplifiers. At the same time, taking the time scale factor $R C$ into account, system (2) after scale transformation can be represented as follows:

$$
\left\{\begin{array}{l}
R C \dot{x}=a x-10 b y z \\
R C \dot{y}=-c y+10 x z+e\left(f+300 g w^{2}\right) x, \\
R C \dot{z}=-\mathrm{d} z+10 x y \\
R C \dot{w}=h x .
\end{array}\right.
$$

A flux-controlled memristor depicted in the dashed box of Figure 15 is applied to construct the chaotic circuit. The analog circuit of system (2) is shown in Figure 15. The state equations can be obtained as follows:

$$
\left\{\begin{array}{l}
C_{1} \dot{v}_{x}=\frac{v_{x}}{R_{1}}-\frac{v_{y} v_{z}}{R_{2}} \\
C_{2} \dot{v}_{y}=-\frac{v_{y}}{R_{3}}+\frac{v_{x} v_{z}}{R_{4}}+\left(\frac{v_{x}}{R_{c}}+\frac{v_{x} v_{w}^{2}}{R_{b}}\right) \\
C_{3} \dot{v}_{z}=-\frac{v_{z}}{R_{5}}+\frac{v_{x} v_{y}}{R_{6}} \\
C_{4} \dot{v}_{w}=\frac{v_{x}}{R_{a}}
\end{array}\right.
$$

where $v_{x}, v_{y}, v_{z}$, and $v_{w}$ are the voltages on capacitors. Compared with (9) and (10), the parameters are taken as follows: $C_{1}=C_{2}=C_{3}=C_{4}=C, R_{1}=(R / a), R_{2}=(R / 10 b)$, $R_{3}=(R / c), \quad R_{4}=(R / 10), \quad R_{5}=(R / d), \quad R_{6}=(R / 10)$, $R_{a}=(R / h), R_{b}=\left(R /\left(e^{*} 3 g^{*} 100\right)\right)$, and $R_{c}=(R / e f)$.

System (2) can generate coexisting infinitely many attractors when the parameters are set as $a=4, b=6, c=20$, $d=5, e=0.01, f=1,3 g=0.1$, and $h=0.1$. Let us take $R=$ $100 \mathrm{k} \Omega$ and $C=10000 \mathrm{nF}$, and the resistance parameters can be obtained as $R_{1}=25 \mathrm{k} \Omega, R_{2}=1.67 \mathrm{k} \Omega, R_{3}=5 \mathrm{k} \Omega$, $R_{4}=10 \mathrm{k} \Omega, \quad R_{5}=20 \mathrm{k} \Omega, \quad R_{6}=10 \mathrm{k} \Omega, \quad R_{a}=1000 \mathrm{k} \Omega$, $R_{b}=1000 \mathrm{k} \Omega$, and $R_{c}=10000 \mathrm{k} \Omega$. Also, the initial voltages of all capacitors are set as $(1 \mathrm{~V}, 0.1 \mathrm{~V}, 0 \mathrm{~V}, 0 \mathrm{~V})$ (initial voltages of all capacitors should be compressed to the $1 / 10$ of original values). According to the above parameter settings, circuit simulation of chaotic attractor can be obtained by PSpice as shown in Figure 16. Similarly, when the initial voltages of all capacitors are set as $(-1 \mathrm{~V}, 0.1 \mathrm{~V}, 0 \mathrm{~V}, 0 \mathrm{~V})$, circuit simulation of chaotic attractor can be obtained by PSpice as shown in Figure 17. Obviously, the chaotic attractors obtained in the analog circuit well verify those shown by numerical simulations.

\section{Conclusion}

A new memristor-based chaotic system with coexisting infinitely many attractors is proposed by using a smooth fluxcontrolled memristor to replace a resistor in the analog circuit of the three-dimensional chaotic system. The system has a line equilibrium and exhibits homogenous and heterogeneous multistabilities. The dynamical behaviors of the system are analyzed by equilibrium point and stability, phase portraits, bifurcation diagrams and Lyapunov exponent spectra, and so on. Compared with general chaotic systems, this chaotic system has some special properties. When the parameters of system are fixed, the chaotic system only generates two kinds of chaotic attractors with different positions in a very large range of initial values and has constant Lyapunov exponent spectra. In addition, the system has discrete bifurcation diagrams, which has not been found in existing chaotic systems. Further, the relationship between the symmetry of the coexisting attractors and the symmetry of initial values in the system is explored and verified by phase portraits and bifurcation diagrams. Finally, the chaotic system is realized by analog circuit, and the numerical simulation results are verified by the simulation results of the analog circuit. The complex dynamical behaviors of the proposed system are very useful for various chaos-based information encryption and secure communication applications.

\section{Data Availability}

The data used to support the findings of this study are included within the article.

\section{Conflicts of Interest}

The authors declare that there no conflicts of interest.

\section{Acknowledgments}

This research was funded by the National Natural Science Foundation of China (nos. 61203004 and 61306142), Natural Science Foundation of Heilongjiang Province (grant no. F201220), and Fundamental Research Funds for the Central Universities (no. 3072019CFG0802).

\section{References}

[1] L. Chua, "Memristor-the missing circuit element," IEEE Transactions on Circuit Theory, vol. 18, no. 5, pp. 507-519, 1971. 
[2] D. B. Strukov, G. S. Snider, D. R. Stewart, and R. S. Williams, "The missing memristor found," Nature, vol. 453, no. 7191, pp. 80-83, 2008.

[3] S. Hamdioui, M. Taouil, and N. Z. Haron, "Testing open defects in memristor-based memories," IEEE Transactions on Computers, vol. 64, no. 1, pp. 247-259, 2015.

[4] S. Kumar, J. P. Strachan, and R. S. Williams, "Chaotic dynamics in nanoscale $\mathrm{NbO}_{2}$ Mott memristors for analogue computing," Nature, vol. 548, no. 7667, pp. 318-321, 2017.

[5] Z. Wang, S. Joshi, S. E. Savel'ev et al., "Memristors with diffusive dynamics as synaptic emulators for neuromorphic computing," Nature Materials, vol. 16, no. 1, pp. 101-108, 2017.

[6] S. Wen, Z. Zeng, M. Z. Q. Chen, and T. Huang, "Synchronization of switched neural networks with communication delays via the event-triggered control," IEEE Transactions on Neural Networks and Learning Systems, vol. 28, pp. 23342343, 2017.

[7] G. Zhang, F. Wu, T. Hayat, and J. Ma, "Selection of spatial pattern on resonant network of coupled memristor and Josephson junction," Communications in Nonlinear Science and Numerical Simulation, vol. 65, pp. 79-90, 2018.

[8] L. L. Huang, Z. F. Zhang, J. H. Xiang, and S. M. Wang, "A new $4 \mathrm{D}$ chaotic system with two-wing, four-wing, and coexisting attractors and its circuit simulation," Complexity, vol. 2019, Article ID 5803506, 13 pages, 2019.

[9] C. Li, X. Wang, and G. Chen, "Diagnosing multistability by offset boosting," Nonlinear Dynamics, vol. 90, no. 2, pp. 1335-1341, 2017.

[10] V.-T. Pham, A. Akgul, C. Volos, S. Jafari, and T. Kapitaniak, "Dynamics and circuit realization of a no-equilibrium chaotic system with a boostable variable," AEU-International Journal of Electronics and Communications, vol. 78, pp. 134-140, 2017.

[11] L. Wang, "3-Scroll and 4-scroll chaotic attractors generated from a new 3-D quadratic autonomous system," Nonlinear Dynamics, vol. 56, no. 4, pp. 453-462, 2009.

[12] G. Grassi and S. Mascolo, "A system theory approach for designing cryptosystems based on hyperchaos," IEEE Transactions on Circuits and Systems I: Fundamental Theory and Applications, vol. 46, no. 9, pp. 1135-1138, 1999.

[13] B. Wang, F. C. Zou, and J. Cheng, "A memristor-based chaotic system and its application in image encryption," Optik, vol. 154, pp. 538-544, 2018.

[14] M. F. Hassan, "A new approach for secure communication using constrained hyperchaotic systems," Applied Mathematics and Computation, vol. 246, pp. 711-730, 2014.

[15] R. L. Filali, M. Benrejeb, and P. Borne, "On observer-based secure communication design using discrete-time hyperchaotic systems," Communications in Nonlinear Science and Numerical Simulation, vol. 19, no. 5, pp. 1424-1432, 2014.

[16] Y. Zhang, M. Guo, G. Dou, Y. Li, and G. Chen, "A physical SBT-memristor-based Chua's circuit and its complex dynamics," Chaos: An Interdisciplinary Journal of Nonlinear Science, vol. 28, no. 8, Article ID 083121, 2018.

[17] M. Itoh and L. O. Chua, "Memristor oscillators," International Journal of Bifurcation and Chaos, vol. 18, no. 11, pp. 31833206, 2008.

[18] B. C. Bao, Z. Liu, and J. P. Xu, "Transient chaos in smooth memristor oscillator," Chinese Physics B, vol. 19, no. 3, Article ID 030510, 2010.

[19] C. Wang, H. Xia, and L. Zhou, "A memristive hyperchaotic multiscroll jerk system with controllable scroll numbers," International Journal of Bifurcation and Chaos, vol. 27, no. 6, Article ID 1750091, 2017.
[20] N. Stankevich and E. Volkov, "Multistability in a three-dimensional oscillator: tori, resonant cycles and chaos," Nonlinear Dynamics, vol. 94, no. 4, pp. 2455-2467, 2018.

[21] K. Rajagopal, S. Jafari, A. Karthikeyan, A. Srinivasan, and B. Ayele, "Hyperchaotic memcapacitor oscillator with infinite equilibria and coexisting attractors," Circuits, Systems, and Signal Processing, vol. 37, no. 9, pp. 3702-3724, 2018.

[22] S. Zhang, Y. Zeng, Z. Li, M. Wang, X. Zhang, and D. Chang, "A novel simple no-equilibrium chaotic system with complex hidden dynamics," International Journal of Dynamics and Control, vol. 6, no. 4, pp. 1465-1476, 2018.

[23] F. Yuan, G. Wang, and X. Wang, "Extreme multistability in a memristor-based multi-scroll hyper-chaotic system," Chaos: An Interdisciplinary Journal of Nonlinear Science, vol. 26, no. 7, Article ID 073107, 2016.

[24] L. Zhou, C. Wang, and L. Zhou, "Generating four-wing hyperchaotic attractor and two-wing, three-wing, and fourwing chaotic attractors in $4 \mathrm{D}$ memristive system," International Journal of Bifurcation and Chaos, vol. 27, no. 2, Article ID 1750027, 2017.

[25] Q. Xu, Y. Lin, B. Bao, and M. Chen, "Multiple attractors in a non-ideal active voltage-controlled memristor based Chua's circuit," Chaos, Solitons \& Fractals, vol. 83, pp. 186-200, 2016.

[26] J. Kengne, Z. Njitacke Tabekoueng, V. Kamdoum Tamba, and A. Nguomkam Negou, "Periodicity, chaos, and multiple attractors in a memristor-based Shinriki's circuit," Chaos: An Interdisciplinary Journal of Nonlinear Science, vol. 25, no. 10, Article ID 103126, 2015.

[27] B. C. Bao, H. Bao, N. Wang, M. Chen, and Q. Xu, "Hidden extreme multistability in memristive hyperchaotic system," Chaos, Solitons \& Fractals, vol. 94, pp. 102-111, 2017.

[28] H. Bao, T. Jiang, K. B. Chu, M. Chen, Q. Xu, and B. C. Bao, "Memristor-based canonical Chua's circuit: extreme multistability in voltage-current domain and its controllability in flux-charge domain," Complexity, vol. 2018, Article ID 5935637, 13 pages, 2018.

[29] M. Chen, M. Sun, B. Bao, H. Wu, Q. Xu, and J. Wang, "Controlling extreme multistability of memristor emulatorbased dynamical circuit in flux-charge domain," Nonlinear Dynamics, vol. 91, no. 2, pp. 1395-1412, 2018.

[30] G. Y. Wang, C. B. Shi, X. W. Wang, and F. Yuan, "Coexisting oscillation and extreme multistability for a memcapacitorbased circuit," Mathematical Problems in Engineering, vol. 2017, Article ID 6504969, 13 pages, 2017.

[31] H. Bao, N. Wang, B. Bao, M. Chen, P. Jin, and G. Wang, "Initial condition-dependent dynamics and transient period in memristor-based hypogenetic jerk system with four line equilibria," Communications in Nonlinear Science and $\mathrm{Nu}$ merical Simulation, vol. 57, pp. 264-275, 2018.

[32] F. Yuan, Y. Deng, Y. Li, and G. Wang, "The amplitude, frequency and parameter space boosting in a memristormeminductor-based circuit," Nonlinear Dynamics, vol. 96, no. 1, pp. 389-405, 2019.

[33] W. Liu and G. Chen, "A new chaotic system and its generation," International Journal of Bifurcation and Chaos, vol. 13, no. 1, pp. 261-267, 2003.

[34] W. Liu and G. Chen, "Can a three-dimensional smooth autonomous quadratic chaotic system generate a single fourscroll attractor?" International Journal of Bifurcation and Chaos, vol. 14, no. 4, pp. 1395-1403, 2004. 